JOURNAL OF

SYMPLECTIC GEOMETRY

Volume 8, Number 1, 95-118, 2010

\title{
COISOTROPIC SUBMANIFOLDS, LEAF-WISE FIXED POINTS, AND PRESYMPLECTIC EMBEDDINGS
}

\author{
FABian ZiLtener
}

Let $(M, \omega)$ be a geometrically bounded symplectic manifold, $N \subseteq$ $M$ a closed, regular (i.e., "fibering") coisotropic submanifold, and $\varphi$ : $M \rightarrow M$ a Hamiltonian diffeomorphism. The main result of this article is that the number of leaf-wise fixed points of $\varphi$ is bounded below by the sum of the $\mathbb{Z}_{2}$-Betti numbers of $N$, provided that the Hofer distance between $\varphi$ and the identity is small enough and the pair $(N, \varphi)$ is non-degenerate. The bound is optimal if there exists a $\mathbb{Z}_{2}$-perfect Morse function on $N$. A version of the Arnol'd-Givental conjecture for coisotropic submanifolds is also discussed. As an application, I prove a presymplectic non-embedding result.

\section{CONTEnTs}

1. Main results $\quad 96$

1.1. Leaf-wise fixed points 96

1.2. Idea of proof of Theorem $1.1 \quad 98$

1.3. Discussion of optimality 98

1.4. Arnol'd-Givental conjecture (AGC) for coisotropic submanifolds

1.5. An application $\quad 99$

1.6. Further research 100

1.7. Related results 100

1.8. Organization of the article 102

2. Background 103

2.1. Notation, manifolds 103

2.2. Foliations, regularity and linear holonomy 103

2.3. Presymplectic manifolds and symplectic quotients 104 


\subsection{Coisotropic submanifolds, leaf-wise fixed points and non-degeneracy}

\section{Main results}

1.1. Leaf-wise fixed points. Let $(M, \omega)$ be a symplectic manifold. We denote by $\operatorname{Ham}(M, \omega)$ the group of Hamiltonian diffeomorphisms (generated by time-dependent functions with arbitrary support). Let $\varphi \in \operatorname{Ham}(M, \omega)$ and $N \subseteq M$ be a coisotropic submanifold. For $x \in N$ we denote by $N_{x}:=N_{x}^{\omega} \subseteq N$ the isotropic leaf through $x$. A leaf-wise fixed point of $\varphi$ is by definition a point $x \in N$ such that $\varphi(x) \in N_{x}$. We denote by $\operatorname{Fix}(\varphi, N):=\operatorname{Fix}(\varphi, N, \omega)$ the set of such points. The first main result of this article addresses the following question:

Question A. Provided that $\varphi$ is close to the identity in a suitable sense, what lower bound on the number $|\operatorname{Fix}(\varphi, N)|$ is there?

Note that if $N=M$ then $N_{x}=\{x\}$, for every $x \in N$, and hence $\operatorname{Fix}(\varphi, N)$ is the set $\operatorname{Fix}(\varphi)$ of ordinary fixed points of $\varphi$. In the other extreme case where $\operatorname{dim} N=\operatorname{dim} M / 2$, the submanifold $N$ is Lagrangian, and we have $\operatorname{Fix}(\varphi, N)=N \cap \varphi^{-1}(N)$, provided that $N$ is connected. In order to state the first main result, we denote by $A(M, \omega, N)$ the minimal area of $(M, \omega, N)$ (see (2.6) below) and by $d:=d^{M, \omega}$ the Hofer distance. We call $N$ regular iff its isotropic leaf relation is a closed subset and a submanifold of $N \times N$. Assuming that $N$ is closed (i.e., compact and without boundary), this means that there exists a manifold structure on the set $N_{\omega}$ of isotropic leaves of $N$ such that the canonical projection $\pi_{N}: N \rightarrow N_{\omega}$ is a smooth (locally trivial) fiber bundle. For the definitions of (geometric) boundedness of $(M, \omega)$ and non-degeneracy for $(N, \varphi)$ see Section 2 . The former is a mild condition on $(M, \omega)$, and the latter naturally generalizes the usual non-degeneracy in the cases $N=M$ and $\operatorname{dim} N=\operatorname{dim} M / 2$. For each topological space $X$ and integer $i$ we denote by $b_{i}(X):=\operatorname{dim} H_{i}\left(X, \mathbb{Z}_{2}\right)$ the $i$ th Betti number of $X$ with coefficients in $\mathbb{Z}_{2}$.

Theorem 1.1. Let $(M, \omega)$ be a (geometrically) bounded symplectic manifold and $N \subseteq M$ a closed, regular coisotropic submanifold. Then there exists a constant $C \in(0, \infty]$ such that $C \geq A(M, \omega, N)$ and the following holds. If 
$\varphi \in \operatorname{Ham}(M, \omega)$ is such that $(N, \varphi)$ is non-degenerate and $d(\varphi, \mathrm{id})<C$ then

$$
|\operatorname{Fix}(\varphi, N)| \geq \sum_{i=0}^{\operatorname{dim} N} b_{i}(N)
$$

If codim $N \neq 0,1, \operatorname{dim} M / 2$ then this theorem appears to be the first result implying that $|\operatorname{Fix}(\varphi, N)| \geq 2$, without assuming that $\varphi$ is $C^{1}$-close to the identity. It generalizes a result for the case $\operatorname{dim} N=\operatorname{dim} M / 2$, which is due to Chekanov $[\mathbf{C h}]$. The bound (1.1) is sharp, provided that there exists a $\mathbb{Z}_{2}$-perfect Morse function on $N$, see Theorem 1.2 below.

Examples. A large class of examples of regular coisotropic submanifolds is given as follows. Let $(M, \omega)$ be a symplectic manifold and $G$ a compact, connected Lie group with Lie algebra $\mathfrak{g}$. We fix a Hamiltonian action of $G$ on $M$ and an (equivariant) moment map $\mu: M \rightarrow \mathfrak{g}^{*}$. Assume that $\mu$ is proper and the action of $G$ on $N:=\mu^{-1}(0) \subseteq M$ is free. Then $N$ is a closed, regular coisotropic submanifold. As a concrete example, let $0<k \leq n$ be integers, and consider $M:=\mathbb{C}^{k \times n}$ with the standard symplectic structure $\omega:=\omega_{0}$ and the action of the unitary group $G:=U(k)$ on $\mathbb{C}^{k \times n}$ by multiplication from the left. A moment map for this action is given by $\mu(\Theta):=\frac{i}{2}\left(\mathbf{1}-\Theta \Theta^{*}\right)$, and $N=\mu^{-1}(0)$ is the Stiefel manifold $V(k, n)$. The triple $(M, \omega, N)$ satisfies the hypotheses in Theorem 1.1. Furthermore, we have $A\left(\mathbb{C}^{k \times n}, \omega_{0}, V(k, n)\right)=\pi$ (see Proposition 1.3 below), and $\sum_{i=0}^{\operatorname{dim} V(k, n)} b_{i}(V(k, n))=2^{k}$. (The latter follows for example from [GHV, Theorem I, p. 224], and the fact that the integral cohomology of $V(k, n)$ is torsion-free.) Let $\varphi \in \operatorname{Ham}\left(\mathbb{C}^{k \times n}, \omega_{0}\right)$ be such that $(V(k, n), \varphi)$ is non-degenerate and $d(\varphi$, id $)<\pi$. Then Theorem 1.1 implies that $|\operatorname{Fix}(\varphi, V(k, n))| \geq 2^{k}$.

By Theorem 1.2 below this bound is sharp, since there exists a $\mathbb{Z}_{2}$-perfect Morse function on $V(k, n)$. (See [TT], the remarks after Ex. 3.14 on p. 197 and the definition of tautness on p. 182.) In this example, the condition $C \geq$ $A(M, \omega, N)$ in Theorem 1.1 is also sharp, in the sense that the conclusion of the theorem is wrong if we choose $C>\pi$, see Proposition 1.3. Note that in the case $k=1$ we obtain an improvement of a result by Hofer [Ho, Proposition 1.4]. That result states that $\operatorname{Fix}\left(\varphi, S^{2 n-1}\right) \neq \emptyset$, provided that $d_{\mathrm{c}}(\varphi, \mathrm{id}) \leq \pi$. Here $d_{\mathrm{c}}$ denotes the compactly supported Hofer distance.

Another family of examples of regular coisotropic submanifolds arises as follows. Let $(X, \sigma)$ be a closed symplectic manifold, $\pi: E \rightarrow X$ a closed smooth fiber bundle and $H \subseteq T E$ a horizontal subbundle. We define $V^{*} E$ to be the vertical cotangent bundle of $E$. Its fiber over a point $e \in E$ is $T_{e}^{*} E_{\pi(e)}$. We denote the zero-section of this bundle by $N$. Furthermore, we define a closed two-form on $V^{*} E$ as follows. We denote by $\pi_{X}: V^{*} E \rightarrow X$ the canonical projection, by $\omega_{\text {can }}$ the canonical symplectic form on $T^{*} E$ and by $\operatorname{pr}_{e}^{H}: T_{e} E \rightarrow T_{e} E_{\pi(e)}$ the linear projection along the subspace $H_{e} \subseteq T_{e} E$, 
for $e \in E$. We define

$$
\iota_{H}: V^{*} E \rightarrow T^{*} E, \quad \iota_{H}(e, \alpha):=\left(e, \alpha \circ \operatorname{pr}_{e}^{H}\right), \quad \Omega_{\sigma, H}:=\pi_{X}^{*} \sigma+\iota_{H}^{*} \omega_{\text {can }} .
$$

Then $\Omega_{\sigma, H}$ is a closed two-form on $V^{*} E$. Furthermore, by Proposition 3.2 in [Ma], there exists an open neighborhood $M$ of the zero-section $N \subseteq V^{*} E$ on which $\Omega_{\sigma, H}$ is non-degenerate. We fix such an $M$. Then the submanifold $N \subseteq M$ is regular coisotropic (see Proposition A.2 below). Assume now that the base manifold $X$ is symplectically aspherical, i.e., $\int_{S^{2}} u^{*} \sigma=0$, for every $u \in C^{\infty}\left(S^{2}, X\right)$. Then $A\left(M, \Omega_{\sigma, H}, N\right)=\infty$ (see again Proposition A.2 below). So in this case the only possible constant $C$ as in Theorem 1.1 is $\infty$, and hence the condition $d(\varphi$, id $)<C$ is vacuous.

1.2. Idea of proof of Theorem 1.1. Assume that the hypotheses of Theorem 1.1 are satisfied. The strategy of the proof is to find a Lagrangian embedding of $N$ into a suitable symplectic manifold, and then apply the Main Theorem in $[\mathbf{C h}]$. Since $N$ is regular, the set of isotropic leaves $N_{\omega}$ carries canonical smooth and symplectic structures $\mathcal{A}_{N, \omega}$ and $\omega_{N}$. We define

$$
\begin{gathered}
\widetilde{M}:=M \times N_{\omega}, \quad \widetilde{\omega}:=\omega \oplus\left(-\omega_{N}\right), \\
\iota_{N}: N \rightarrow \widetilde{M}, \quad \iota_{N}(x):=\left(x, N_{x}\right), \quad \widetilde{N}:=\iota_{N}(N) .
\end{gathered}
$$

Then $\iota_{N}$ is an embedding of $N$ into $\widetilde{M}$ that is Lagrangian with respect to the symplectic form $\widetilde{\omega}$ on $\widetilde{M}$ (see Lemma 3.2 below). In order to satisfy the hypotheses of Chekanov's result, the inequality $A(M, \omega, N) \leq A(\widetilde{M}, \widetilde{\omega}, \widetilde{N})$ is crucial. It follows from Key Lemma 3.4 below. The idea of its proof is that given a smooth map $\widetilde{u}=\left(v, w^{\prime}\right): \mathbb{D} \rightarrow \widetilde{M}=M \times N_{\omega}$ such that $\widetilde{u}\left(S^{1}\right) \subseteq \widetilde{N}$, we may lift $w^{\prime}$ to a map $w:[0,1] \times S^{1} \rightarrow N$ and concatenate this with $v$. We thus obtain a map $u: \mathbb{D} \rightarrow M$ with boundary on an isotropic leaf, satisfying $\int u^{*} \omega=\int \widetilde{u}^{*} \widetilde{\omega}$. The method described here generalizes a standard way of reducing the case $\operatorname{dim} N=\operatorname{dim} M$ to the Lagrangian case, see e.g., [Fl].

1.3. Discussion of optimality. Let $M$ be a manifold and $f: M \rightarrow \mathbb{R}$ a Morse function. We denote by Crit $f \subseteq M$ the set of critical points of $f$. Recall that $f$ is called $\mathbb{Z}_{2}$-perfect iff $\mid$ Crit $f \mid=\sum_{i=0}^{\operatorname{dim}} M_{i} b_{i}(M)$. The next result implies that the estimate (1.1) is sharp if there exists a $\mathbb{Z}_{2}$-perfect Morse function on the coisotropic submanifold $N$. It actually shows that in this case (1.1) is sharp, even if the condition $d(\varphi, \mathrm{id})<C$ is replaced by the much stronger condition that $\varphi$ is $C^{1}$-close to the identity. We denote by $\operatorname{Ham}_{\mathrm{c}}(M, \omega)$ the group of Hamiltonian diffeomorphisms generated by compactly supported time-dependent functions.

Theorem 1.2. Let $(M, \omega)$ be a symplectic manifold, $N \subseteq M$ a closed regular coisotropic submanifold, $f: N \rightarrow \mathbb{R}$ a Morse function and $\mathcal{U} \subseteq \operatorname{Ham}_{\mathrm{c}}(M, \omega)$ a $C^{1}$-neighborhood of id. Then there exists $\varphi \in \mathcal{U}$ such that $(N, \varphi)$ is nondegenerate and $\operatorname{Fix}(\varphi, N)=\operatorname{Crit} f$. 
The proof of this result relies on a normal form theorem for a neighborhood of $N$, due to Marle, and on the fact that fast almost periodic orbits of a vector field are constant. It also uses an estimate for the distance between the initial and the end point of a path $x$ in a foliation, assuming that these points lie in the same leaf, and that $x$ is tangent to a given horizontal distribution.

Examples of manifolds admitting a $\mathbb{Z}_{2}$-perfect Morse function are compact symmetric spaces that admit a symmetric embedding into Euclidian space (e.g., Grassmannians, see [DV, Theorem 1.2, Example 1, p. 7.]), symplectic manifolds that admit a Hamiltonian $S^{1}$-action whose fixed points are isolated (see [GGK, Theorem p. 22]), and simply connected closed manifolds of dimension at least 6 , whose homology with $\mathbb{Z}$-coefficients is torsion-free (see [An, Theorem 4.2.4(ii)]).

The next result shows that for $M:=\mathbb{C}^{k \times n}, \omega:=\omega_{0}$ and the Stiefel manifold $N:=V(k, n)$ the condition $C \geq A(M, \omega, N)$ in Theorem 1.1 is sharp.

Proposition 1.3. We have $A\left(\mathbb{C}^{k \times n}, \omega_{0}, V(k, n)\right)=\pi$. Furthermore, for every $C>\pi$ there exists $\varphi \in \operatorname{Ham}_{\mathrm{c}}\left(\mathbb{C}^{k \times n}, \omega_{0}\right)$ such that $d_{\mathrm{c}}(\varphi, \mathrm{id})<C$ and $\varphi(V(k, n)) \cap V(k, n)=\emptyset$.

1.4. Arnol'd-Givental conjecture (AGC) for coisotropic submanifolds. Recall that a map from a set to itself is called an involution iff applying it twice yields the identity. Furthermore, a diffeomorphism $\psi$ from a symplectic manifold $(M, \omega)$ to itself is called anti-symplectic iff $\psi^{*} \omega=-\omega$. The following conjecture naturally generalizes the usual (Lagrangian) AGC, which corresponds to the case in which $M_{1}$ is a point. (See for example $[\mathbf{F r}]$, where it is assumed that $M$ is compact.)

Conjecture. Let $\left(M_{i}, \omega_{i}\right), i=1,2$ be symplectic manifolds, with $M_{1}$ closed, and let $L \subseteq M_{2}$ be a closed Lagrangian submanifold. Consider the product $M:=M_{1} \times M_{2}$ with the symplectic structure $\omega:=\omega_{1} \oplus \omega_{2}$, and let $N:=$ $M_{1} \times L$. Assume that there exists an anti-symplectic involution $\psi: M_{2} \rightarrow M_{2}$ such that $\operatorname{Fix}(\psi)=L$. Let $\varphi \in \operatorname{Ham}(M, \omega)$ be such that the pair $(N, \varphi)$ is non-degenerate. Then inequality (1.1) holds.

Proposition 1.4. If the Lagrangian $A G C$ is true then the same holds for the above Conjecture.

1.5. An application. We say that a presymplectic manifold $\left(M^{\prime}, \omega^{\prime}\right)$ (see Section 2) embeds into a presymplectic manifold $(M, \omega)$ iff there exists an embedding $\psi: M^{\prime} \rightarrow M$ such that $\psi^{*} \omega=\omega^{\prime}$. The following question generalizes the symplectic and Lagrangian embedding problems.

Question B. Given two presymplectic manifolds, does one of them embed into the other one? 
Note that in the case $\operatorname{dim} M^{\prime}+\operatorname{corank} \omega^{\prime}>\operatorname{dim} M+\operatorname{corank} \omega$ there does not even exist any immersion $\psi: M^{\prime} \rightarrow M$ satisfying $\psi^{*} \omega=\omega^{\prime}$. The next result is concerned with the "critical case" in which " $>$ " is replaced by "=" above. It is a consequence of Theorem 1.1. A presymplectic manifold $(M, \omega)$ is called regular iff its isotropic leaf relation is a closed subset of $M \times M$ and a submanifold.

Corollary 1.5. Let $(M, \omega)$ be a bounded and aspherical symplectic manifold, and $\left(M^{\prime}, \omega^{\prime}\right)$ a closed, regular presymplectic manifold of corank $\operatorname{dim} M-$ $\operatorname{dim} M^{\prime}$. Assume that every compact subset of $M$ can be displaced in a Hamiltonian way, and that $M^{\prime}$ has a simply connected isotropic leaf. Then $\left(M^{\prime}, \omega^{\prime}\right)$ does not embed into $(M, \omega)$.

Examples. As an example, let $(X, \sigma)$ and $\left(X^{\prime}, \sigma^{\prime}\right)$ be symplectic manifolds, the former bounded and aspherical and the latter closed. Let $F$ be a closed simply connected manifold. Assume that $\operatorname{dim} X+2=\operatorname{dim} X^{\prime}+2 \operatorname{dim} F$. Then the hypotheses of Corollary 1.5 are satisfied with

$$
M:=X \times \mathbb{R}^{2}, \quad \omega:=\sigma \oplus \omega_{0}, \quad M^{\prime}:=X^{\prime} \times F, \quad \omega^{\prime}:=\sigma^{\prime} \oplus 0 .
$$

As a more specific example, let $\left(X^{\prime}, \sigma^{\prime}\right)$ be a closed aspherical symplectic manifold, and $k \geq 2$ and $0 \leq \ell \leq k$ be integers. We define

$$
(M, \omega):=\left(X^{\prime} \times \mathbb{R}^{2(k-\ell)} \times \mathbb{R}^{\ell}, \sigma^{\prime} \oplus \omega_{0} \oplus 0\right), \quad\left(M^{\prime}, \omega^{\prime}\right):=\left(X^{\prime} \times S^{k}, \sigma^{\prime} \oplus 0\right) .
$$

Then $\left(M^{\prime}, \omega^{\prime}\right)$ does not embed into $(M, \omega)$. To see this, observe that every embedding of $\left(M^{\prime}, \omega^{\prime}\right)$ into $(M, \omega)$ gives rise to an embedding of $\left(M^{\prime}, \omega^{\prime}\right)$ into $\left(X^{\prime} \times \mathbb{R}^{2 k}, \sigma^{\prime} \oplus \omega_{0}\right)$, by composition with the canonical inclusion $M \rightarrow$ $X^{\prime} \times \mathbb{R}^{2 k}$. Hence the statement follows from Corollary 1.5.

However, in this example there exists an embedding $\psi: M^{\prime} \rightarrow M$ such that $\psi^{*}[\omega]=\left[\omega^{\prime}\right]$, provided that $\ell<k$. We may for example choose any embedding $\iota: S^{k} \rightarrow \mathbb{R}^{2(k-\ell)} \times \mathbb{R}^{\ell}$ and define $\psi:=\mathrm{id}_{X^{\prime}} \times \iota$. Furthermore, if $\ell=0$ then there exists an immersion $\psi: M^{\prime} \rightarrow M$ satisfying $\psi^{*} \omega=\omega^{\prime}$. To see this, note that the Whitney map

$$
f: S^{k} \subseteq \mathbb{R} \times \mathbb{R}^{k} \rightarrow \mathbb{R}^{2 k} \cong \mathbb{C}^{k}, \quad f(a, x):=(1+a i) x
$$

is a Lagrangian immersion (see [ACL, Example I.4.3, p. 17.]). The map $\psi:=\operatorname{id}_{X^{\prime}} \times f$ has the desired properties.

1.6. Further research. A further direction of research is to replace the condition $d(\varphi, \mathrm{id})<C$ by a suitable monotonicity assumption. This requires a definition of a Maslov map for the triple $(M, \omega, N)$. In a forthcoming article [Zi2] I give such a definition.

1.7. Related results. In the extreme cases $N=M$ and $\operatorname{dim} N=\operatorname{dim} M / 2$ Question A has been investigated a lot. For some references, see for example [MS, Sec. 9.1., p. 277] and [Gin, Sec. 1.1. p. 112]. If $(M, \omega)$ is a closed 
symplectic manifold, and $\varphi \in \operatorname{Ham}(M, \omega)$ is such that every $x \in \operatorname{Fix}(\varphi)$ is non-degenerate then Arnol'd $[\mathbf{A r}]$ conjectured that $|\operatorname{Fix}(\varphi)| \geq \mid$ Crit $f \mid$ for every Morse function $f: M \rightarrow \mathbb{R}$.

The general coisotropic case was first considered by Moser. He proved that $|\operatorname{Fix}(\varphi, N)| \geq 2$ if $M$ is simply connected, $\omega$ is exact and $d^{C^{1}}(\varphi$, id) is sufficiently small, see the theorem on p. 19 in [Mos]. (In fact, he showed that $|\operatorname{Fix}(\varphi, N)|$ is bounded below by the Lusternik-Schnirelmann category of $N$, see Proposition 5, p. 31 in [Mos].) Banyaga [Ba] removed the simply connectedness and exactness conditions. Because of the $C^{1}$-closeness condition these are local results. Global results were first obtained by Ekeland and Hofer $\left[\mathbf{E H}, \mathbf{H o}\right.$. For $N$ a closed connected hypersurface in $\mathbb{R}^{2 n}$ of restricted contact type they gave several criteria under which $\operatorname{Fix}(\varphi, N) \neq \emptyset$, allowing for interesting cases in which $d^{C^{1}}(\varphi$, id) is big. For example, in Theorem 1.6 in $[\mathbf{H o}]$ it is assumed that the compactly supported Hofer distance $d_{\mathrm{c}}(\varphi, \mathrm{id})$ is bounded above by the Ekeland-Hofer capacity $c_{\mathrm{EH}}(N)$. Recall here that a coisotropic submanifold $N \subseteq M$ of codimension $k$ is said to be of contact type iff there exist one-forms $\alpha_{1}, \ldots, \alpha_{k}$ on $N$ such that $d \alpha_{i}=\omega$, for $i=1, \ldots, k$, and $\left.\alpha_{1} \wedge \cdots \wedge \alpha_{k} \wedge \omega\right|_{N} ^{n-k}$ does not vanish anywhere on $N$. Here $\left.\omega\right|_{N}$ denotes the pullback of $\omega$ under the inclusion of $N$ into $M . N$ is said to be of restricted contact type iff the $\alpha_{i}$ 's extend to global primitives of $\omega$. Dragnev [Dr, Theorem 1.3] proved a similar result for general codimension of $N$, replacing $c_{\mathrm{EH}}(N)$ by the Floer Hofer capacity of $N$, and assuming that $N$ is only of contact type.

Generalizing in another direction, Ginzburg proved a version of Hofer's result for subcritical Stein manifolds, replacing $c_{\mathrm{EH}}$ by some homological capacity $c_{\text {hom }}$ (see [Gin, Theorem 2.9, p. 122]). This result in turn was recently extended by Gürel $[\mathbf{G} \ddot{\mathbf{u}}]$ to the coisotropic case (with $c_{\text {hom }}$ replaced by some constant depending on $N)$. For general codimension of $N$, Ginzburg observed that $\operatorname{Fix}(\varphi, N) \neq \emptyset$ if the isotropic foliation of $N$ is a fibration (i.e., $N$ is regular) and " $\varphi$ is not far from id in a suitable sense", see [Gin, Example 1.3, p. 113]. His argument is based on the fact that in this case the leaf relation is a Lagrangian submanifold of the product $M \times M$, equipped with the symplectic form $\omega \oplus(-\omega)$. Lately, Albers and Frauenfelder proved that $\operatorname{Fix}(\varphi, N) \neq \emptyset$, if $(M, \omega)$ is convex at infinity, $N \subseteq M$ is a closed hypersurface of restricted contact type, and $d_{\mathrm{c}}(\varphi, \mathrm{id})<A(M, \omega, N)$. If in addition the Rabinowitz action functional of the Hamiltonian function generating $\varphi$ is Morse, then they showed that $|\operatorname{Fix}(\varphi, N)| \geq \sum_{i} b_{i}(N)$ (see Theorems A and $\mathrm{B}$ in $[\mathbf{A F}])$. A problem related to Question $\mathrm{A}$ is to find a lower bound on the displacement energy of a coisotropic submanifold. Recent work on this problem other than the one already mentioned has been carried out by Kerman $[\mathbf{K e}]$.

Note that regularity of $N$ and the contact-type condition do not imply each other. For example, every Lagrangian submanifold is regular. However, 
if $N$ is a closed connected Lagrangian submanifold of contact type then it is a torus, see for example [Gin, Example 2.2 (iv), p. 118]. On the other hand, consider $(M, \omega):=\left(\mathbb{C}^{2}, \omega_{0}\right)$, fix an irrational number $a>0$, and define $H: \mathbb{C}^{2} \rightarrow \mathbb{R}$ by $H(z, w):=|z|^{2}+|w|^{2} / a$. Then the ellipsoid $N:=$ $H^{-1}(1) \subseteq M$ is a hypersurface of restricted contact type, since the region bounded by $N$ is convex. However, the only compact isotropic leaves are the circles $\left\{\left.(z, 0)|| z\right|^{2}=1\right\}$ and $\left\{\left.(0, w)|| w\right|^{2}=a\right\}$. (The leaves are the integral curves of the Hamiltonian vector field of $H$.) Hence $N$ is not regular. Note that "restricted contact type" is a global condition on $(M, \omega, N)$, whereas regularity is a condition only on $\left(N,\left.\omega\right|_{N}\right)$.

If $N$ is of restricted contact type then it is stable (see Definition 2.1 p. 117 in [Gin]). Regularity and stability can be seen as "dual" conditions in the following sense. Namely, $\left(N,\left.\omega\right|_{N}\right)$ is regular if and only if it fibers into isotropic submanifolds, whereas it is stable if and only if some neighborhood of $N$ fibers as a family of coisotropic submanifolds containing $N$, see [Gin, Proposition 2.6, p. 120]. Note also that Ginzburg constructed a closed hypersurface $N \subseteq \mathbb{R}^{2 n}$ without any closed characteristic, see [Gin, Example 7.2, p. 158]. It follows that $N$ is not regular and $A\left(\mathbb{R}^{2 n}, \omega_{0}, N\right)=\infty$. By the latter the conclusion of Theorem 1.1 fails. Furthermore, work in progress by Gürel shows that there exists a closed hypersurface $N \subseteq \mathbb{R}^{2 n}$ such that $A\left(\mathbb{R}^{2 n}, \omega_{0}, N\right)=\infty$, and for every $\varepsilon>0$ there exists $\varphi \in \operatorname{Ham}(M, \omega)$ satisfying $\operatorname{Fix}(\varphi, N)=\emptyset$ and $d_{\mathrm{c}}(\varphi, \mathrm{id})<\varepsilon$. This shows that one may not completely drop the regularity or stability condition on $N$ if one wants to prove existence of a leaf-wise fixed point.

Let now $M, \omega, M^{\prime}$ and $\omega^{\prime}$ be as in the hypothesis of Corollary 1.5. Assume that $(M, \omega)$ is the product of some bounded symplectic manifold with $\left(\mathbb{R}^{2}, \omega_{0}\right)$ and that $M^{\prime}$ is simply connected. Then the statement of the corollary follows from the comments after Example 2.2.8. on pp. 288 and 289 in $[\mathbf{A L P}]$, using Proposition A.1 and Lemma A.5 below. Like the proof of Corollary 1.5, that argument is based on the fact that the image of the map $\iota_{N}$ defined in (1.3) is a Lagrangian submanifold of $M \times N_{\omega}$. However, since it does not involve the Key Lemma 3.4, the assumption that $M^{\prime}$ is simply connected is needed there. On the other hand, if $\omega$ is exact then the statement of Corollary 1.5 can be deduced from Example 1.7, p. 115 in [Gin], using again Proposition A.1 and Lemma A.5. Furthermore, if the presymplectic manifold $\left(M^{\prime}, \omega^{\prime}\right)$ is stable then a similar non-embedding result can be deduced from Theorem 2.7(ii), p. 121 in [Gin].

1.8. Organization of the article. Section 2 contains some background on foliations, presymplectic manifolds, coisotropic submanifolds and leaf-wise fixed points. In this section, the linear holonomy of a foliation along a path in a leaf, and based on this, non-degeneracy of a pair $(N, \varphi)$, are defined. In Section 3 Chekanov's theorem is restated (Theorem 3.1), and the relevant properties of $\widetilde{M}, \widetilde{\omega}, \iota_{N}$ and $\widetilde{N}$ (as in (1.2), (1.3)) are established (Lemmas 
3.2 and 3.4). Based on this, the main results are proven in Section 4. The Appendix contains some auxiliary results.

\section{Background}

2.1. Notation, manifolds. We denote by $\mathbb{D}, S^{1} \subseteq \mathbb{R}^{2}$ the closed unit disk and the unit circle, and for $r>0$ by $B_{r}$ and $\bar{B}_{r} \subseteq \mathbb{R}^{2}$ the open and closed balls of radius $r$. Let $M$ be a set. By a smooth structure on $M$ we mean a maximal smooth $\left(C^{\infty}\right)$ atlas $\mathcal{A}$ of charts $\varphi: U \subseteq M \rightarrow \mathbb{R}^{n}$. (Hence $M$ does not have any boundary.) We denote by $C_{\mathrm{c}}^{\infty}(M, \mathbb{R})$ the space of compactly supported smooth functions on $M$. We call $(M, \mathcal{A})$ a manifold iff the topology on $M$ induced by $\mathcal{A}$ is Hausdorff and second countable. Submanifolds of $M$ are by definition embedded. If $M$ is equipped with a Riemannian metric then we denote by $|\cdot|, \ell$ and $d$ the induced norm on $T_{x} M$ (for $x \in M$ ) and length and distance functions.

2.2. Foliations, regularity and linear holonomy. Let $M$ be a manifold and $\mathcal{F}$ a foliation on $M$, i.e., a maximal atlas of foliation charts. We denote by $T \mathcal{F} \subseteq T M$ and $N \mathcal{F}:=T M / T \mathcal{F}$ the tangent and normal bundles of $\mathcal{F}$, by $\operatorname{pr}^{\mathcal{F}}: T M \rightarrow N \mathcal{F}$ the canonical projection, by $\mathcal{F}_{x} \subseteq M$ the leaf through a point $x \in M$ and by $R^{\mathcal{F}}:=\left\{(x, y) \in M \times M \mid y \in \mathcal{F}_{x}\right\}$ the leaf relation. For $x \in M$ we write $T_{x} \mathcal{F}:=(T \mathcal{F})_{x}$ and $N_{x} \mathcal{F}:=(N \mathcal{F})_{x}$. We call $\mathcal{F}$ regular iff $R^{\mathcal{F}}$ is a closed subset and submanifold of $M \times M$. We call a distribution $H \subseteq T M\left(\mathcal{F}\right.$-)horizontal iff for every $x \in M$ we have $T_{x} M=H_{x} \oplus T_{x} \mathcal{F}$. Let $F$ be a leaf of $\mathcal{F}, a \leq b$ and $x \in C^{\infty}([a, b], F)$. For the definition of non-degeneracy of a pair $(N, \varphi)$ as in Section 1 we need the notion of linear holonomy of $\mathcal{F}$ along $x$. This is a linear map hol $\mathcal{F}_{x}^{\mathcal{F}}: N_{x(a)} \mathcal{F} \rightarrow N_{x(b)} \mathcal{F}$, whose definition is based on the following result.

Proposition 2.1. Let $M, \mathcal{F}, F, a, b$ and $x$ be as above, $N$ a manifold and $y_{0} \in N$. Then the following statements hold:

(a) For every linear map $T: T_{y_{0}} N \rightarrow T_{x(a)} M$ there exists a map $u \in$ $C^{\infty}([a, b] \times N, M)$ such that

$$
\begin{gathered}
u\left(\cdot, y_{0}\right)=x, \quad \partial_{t} u(t, y) \in T_{u(t, y)} \mathcal{F}, \forall t \in[a, b], y \in N, \\
d(u(a, \cdot))\left(y_{0}\right)=T .
\end{gathered}
$$

(b) Let $u, u^{\prime} \in C^{\infty}([a, b] \times N, M)$ be maps satisfying (2.1), such that

$$
\operatorname{pr}^{\mathcal{F}} d(u(a, \cdot))\left(y_{0}\right)=\operatorname{pr}^{\mathcal{F}} d\left(u^{\prime}(a, \cdot)\right)\left(y_{0}\right) .
$$

Then $\operatorname{pr}^{\mathcal{F}} d(u(b, \cdot))\left(y_{0}\right)=\operatorname{pr}^{\mathcal{F}} d\left(u^{\prime}(b, \cdot)\right)\left(y_{0}\right)$.

This result is proved in the Appendix. We define $N:=N_{x(a)} \mathcal{F}$ and $y_{0}:=$ 0 , and we canonically identify $T_{0}\left(N_{x(a)} \mathcal{F}\right)=N_{x(a)} \mathcal{F}$. We choose a linear 
map $T: N_{x(a)} \mathcal{F} \rightarrow T_{x(a)} M$, such that $\operatorname{pr}^{\mathcal{F}} T=\operatorname{id}_{N_{x(a)} \mathcal{F}}$, and a map $u \in$ $C^{\infty}\left([a, b] \times N_{x(a)} \mathcal{F}, M\right)$ such that (2.1) and (2.2) hold. We define

$$
\operatorname{hol}_{x}^{\mathcal{F}}:=\operatorname{pr}^{\mathcal{F}} d(u(b, \cdot))(0): N_{x(a)} \mathcal{F}\left(=T_{0}\left(N_{x(a)} \mathcal{F}\right)\right) \rightarrow N_{x(b)} \mathcal{F} .
$$

It follows from Proposition 2.1 that this map is well defined. It can be viewed as the linearization of the holonomy of a foliation as defined for example in Sec. 2.1 in the book [MM]. For a given Riemannian metric on $M$, hol $_{x}^{\mathcal{F}}$ corresponds to the holonomy of a Bott connection (as defined, e.g., in Lemma 6.1.5. in $[\mathbf{C C}]$ ) along $x$.

2.3. Presymplectic manifolds and symplectic quotients. By a presymplectic vector space we mean a real vector space $V$ together with a skew-symmetric bilinear form $\omega$. Let $(V, \omega)$ be such a pair. For every linear subspace $W \subseteq V$ we denote by $W^{\omega}:=\{v \in V \mid \omega(v, w)=0, \forall w \in W\}$ its $\omega$-complement. We define corank $\omega:=\operatorname{dim} V^{\omega}$. A presymplectic structure on a manifold $M$ is a closed two-form $\omega$ on $M$, such that corank $\omega_{x}$ does not depend on $x \in M$. This number is called the corank of $\omega$. Let $(M, \omega)$ be a presymplectic manifold. The isotropic distribution $T M^{\omega}=$ $\left\{(x, v) \mid x \in M, v \in T_{x} M^{\omega}\right\} \subseteq T M$ is involutive. Hence by Frobenius' theorem it induces a foliation $\mathcal{F}^{\omega}$ on $M$. We denote by $M_{\omega}$ the set of its leaves. We call $(M, \omega)$ regular iff $\mathcal{F}^{\omega}$ is regular. In this case there are canonical smooth and symplectic structures $\mathcal{A}_{M, \omega}$ (see Lemma A.5) and $\omega_{M}$ on $M_{\omega}$, and the triple $\left(M_{\omega}, \mathcal{A}_{M, \omega}, \omega_{M}\right)$ is called the symplectic quotient of $(M, \omega)$. Let $H \subseteq T M$ be an $\left(\omega\right.$-)horizontal distribution (i.e., $H$ is $\mathcal{F}^{\omega}$-horizontal). This gives rise to a closed two-form $\Omega_{\omega, H}$ on $\left(T M^{\omega}\right)^{*}$, as follows. We denote by $\pi:\left(T M^{\omega}\right)^{*} \rightarrow M$ the canonical projection, and by $\omega_{\text {can }}$ the canonical symplectic form on $T^{*} M$. For $x \in M$ we denote by $\mathrm{pr}_{x}^{H}: T_{x} M \rightarrow T_{x} M^{\omega}$ the projection along $H_{x} \subseteq T_{x} M$. We define

$$
\iota_{H}:\left(T M^{\omega}\right)^{*} \rightarrow T^{*} M, \iota_{H}(x, \alpha):=\left(x, \alpha \circ \operatorname{pr}_{x}^{H}\right), \Omega_{\omega, H}:=\pi^{*} \omega+\iota_{H}^{*} \omega_{\text {can }} .
$$

By a result by Marle there exists an open neighborhood of the zero section on which $\Omega_{\omega, H}$ is non-degenerate, see Proposition 3.2 in $[\mathbf{M a}]$.

2.4. Coisotropic submanifolds, leaf-wise fixed points and nondegeneracy. Let $(M, \omega)$ be a presymplectic manifold and $N \subseteq M$ a coisotropic submanifold. We call $N$ regular iff the presymplectic manifold $\left(N,\left.\omega\right|_{N}\right)$ is regular. Recall that $N_{\omega}$ denotes the set of isotropic leaves of $N$. We define the action spectrum and the minimal area of $(M, \omega, N)$ as

$$
\begin{gathered}
S(M, \omega, N):=\left\{\int_{\mathbb{D}} u^{*} \omega \mid u \in C^{\infty}(\mathbb{D}, M): \exists F \in N_{\omega}: u\left(S^{1}\right) \subseteq F\right\} \\
A(M, \omega, N):=\inf (S(M, \omega, N) \cap(0, \infty)) \in[0, \infty] .
\end{gathered}
$$


Furthermore, we denote hol ${ }^{\omega, N}:=\operatorname{hol}^{\mathcal{F}^{\omega}}$. Assume now that $\omega$ is symplectic, and let $\varphi$ be a diffeomorphism of $M$. We call $(N, \varphi, \omega)$ (or simply $(N, \varphi)$ ) non-degenerate iff the following holds. For $x_{0} \in N$ we denote by $\operatorname{pr}_{x_{0}}$ : $T_{x_{0}} N \rightarrow T_{x_{0}} N /\left(T_{x_{0}} N\right)^{\omega}$ the canonical projection. Let $F \subseteq N$ be an isotropic leaf, and $x \in C^{\infty}([0,1], F)$ a path. Assume that $\varphi(x(0))=x(1)$, and let $v \in T_{x(0)} N \cap T_{x(0)} \varphi^{-1}(N)$ be a vector. Then $v \neq 0$ implies that

$$
\operatorname{hol}_{x}^{\omega, N} \operatorname{pr}_{x(0)} v \neq \operatorname{pr}_{x(1)} d \varphi(x(0)) v .
$$

In the case $N=M$ this condition means that for every $x_{0} \in \operatorname{Fix}(\varphi), 1$ is not an eigenvalue of $\mathrm{d} \varphi\left(x_{0}\right)$. Furthermore, in the case in which $N$ is Lagrangian the condition means that for every connected component $N^{\prime} \subseteq N$ we have $N^{\prime} \pitchfork \varphi\left(N^{\prime}\right)$, i.e., $N^{\prime}$ and $\varphi\left(N^{\prime}\right)$ intersect transversely.

2.5. Geometric boundedness. We call a symplectic manifold $(M, \omega)$ (geometrically) bounded iff there exists an $\omega$-compatible almost complex structure $J$ on $M$ such that the metric $\omega(\cdot, J \cdot)$ is complete with bounded sectional curvature and injectivity radius bounded away from 0 . Examples are closed symplectic manifolds, cotangent bundles of closed manifolds and symplectic vector spaces.

\section{Reduction to the Lagrangian case}

The proof of Theorem 1.1 is based on the following result:

Theorem 3.1. Let $(M, \omega)$ be a bounded symplectic manifold and $L \subseteq M a$ closed Lagrangian submanifold. Then there exists a constant $C \in(0, \infty]$ such that $C \geq A(M, \omega, L)$ (see (2.6)) and the following holds. If $\varphi \in \operatorname{Ham}(M, \omega)$ satisfies $d(\varphi, \mathrm{id})<C$, and $\varphi(L) \pitchfork L$, then

$$
|L \cap \varphi(L)| \geq \sum_{i} b_{i}(L) .
$$

Proof. This follows from the Main Theorem in $[\mathbf{C h}]$ by an elementary argument.

Let now $(M, \omega)$ be a symplectic manifold and $N \subseteq M$ a coisotropic submanifold. Recall that $\pi_{N}: N \rightarrow N_{\omega}$ denotes the canonical projection. We abbreviate $\pi:=\pi_{N}$. We define $\widetilde{M}:=M \times N_{\omega}$, and $\iota_{N}$ and $\widetilde{N}$ as in (1.3). For a map $\varphi: M \rightarrow M$ we define

$$
\widetilde{\varphi}:=\varphi \times \operatorname{id}_{N_{\omega}}: \widetilde{M} \rightarrow \widetilde{M} .
$$

Assume that $N$ is regular. We equip $N_{\omega}$ with the canonical smooth and symplectic structures $\mathcal{A}_{N, \omega}$ and $\omega_{N}$. Furthermore, we define $\widetilde{\omega}:=\omega \oplus\left(-\omega_{N}\right)$. This is a symplectic form on $\widetilde{M}$.

Lemma 3.2. Let $(M, \omega)$ be a symplectic manifold, and $N \subseteq M$ a connected coisotropic submanifold. 
(a) For every map $\varphi: M \rightarrow M$ we have $\widetilde{\varphi} \circ \iota_{N}(\operatorname{Fix}(\varphi, N))=\tilde{N} \cap \widetilde{\varphi}(\tilde{N})$.

Assume now also that $N$ is regular. Then:

(b) The map $\iota_{N}$ is a Lagrangian embedding with respect to $\widetilde{\omega}$.

(c) If $\varphi: M \rightarrow M$ is a diffeomorphism then the pair $(N, \varphi)$ is nondegenerate if and only $\widetilde{\varphi}(\widetilde{N}) \pitchfork \widetilde{N}$.

For the proof of Lemma 3.2 we need the following.

Lemma 3.3. Let $(M, \omega)$ be a symplectic manifold, $N \subseteq M$ a regular coisotropic submanifold, $\varphi: M \rightarrow M$ a diffeomorphism, $F \subseteq N$ a leaf and $x \in C^{\infty}([0,1], F)$ a path. Assume that $x(1)=\varphi \circ x(0)$, and let $v \in T_{x(0)} N \cap T_{x(0)} \varphi^{-1}(N)$. Then (2.7) is equivalent to $\pi_{*} \varphi_{*} v \neq \pi_{*} v$.

Proof of Lemma 3.3. We fix $y \in N$. By Lemma A.5(b) below we have $\operatorname{ker} \mathrm{d} \pi(y)=T_{y} N^{\omega}$. Hence we may define

$$
\Phi_{y}:\left(T_{y} N\right)_{\omega}=T_{y} N / T_{y} N^{\omega} \rightarrow T_{N_{y}}\left(N_{\omega}\right), \quad \Phi_{y}\left(w+T_{y} N^{\omega}\right):=\mathrm{d} \pi(y) w .
$$

This is an isomorphism. Furthermore, $\Phi_{y} \operatorname{pr}_{y}=d \pi(y)$, where $\operatorname{pr}_{y}: T_{y} N \rightarrow$ $\left(T_{y} N\right)_{\omega}$ denotes the canonical projection. Hence Lemma A.5(f) below implies that $\mathrm{d} \pi(x(0))=\Phi_{x(1)} \operatorname{hol}_{x}^{\omega, N} \operatorname{pr}_{x(0)}$. It follows that $\left(\pi_{*} \varphi_{*}-\pi_{*}\right) v=$ $\Phi_{x(1)}\left(\operatorname{pr}_{x(1)} \varphi_{*}-\operatorname{hol}_{x}^{\omega, N} \operatorname{pr}_{x(0)}\right) v$. Since $\Phi_{x(1)}$ is an isomorphism, the statement of Lemma 3.3 follows.

Proof of Lemma 3.2. Statements (a) and (b) follow directly from the definitions. We prove (c). Assume that $x \in \operatorname{Fix}(\varphi, N)$ and denote $\widetilde{x}:=\left(\varphi(x), N_{x}\right)$. Note that by assertion (a) we have $\widetilde{x} \in \widetilde{N} \cap \widetilde{\varphi}(\widetilde{N})$.

Claim 1. $\widetilde{N}$ and $\widetilde{\varphi}(\widetilde{N})$ intersect transversely at $\widetilde{x}$ if and only if

$$
0 \neq v \in T_{x} N \cap T_{x} \varphi^{-1}(N) \Longrightarrow \pi_{*} \varphi_{*} v \neq \pi_{*} v .
$$

Proof of Claim 1. For $y \in N$ we have

$$
T_{\iota_{N}(y)} \tilde{N}=\iota_{N *} T_{y} N=\left\{\left(v, \pi_{*} v\right) \mid v \in T_{y} N\right\} .
$$

Setting $y:=x$, it follows that

$$
T_{\widetilde{x}} \widetilde{\varphi}(\widetilde{N})=\widetilde{\varphi}_{*} \iota_{N *} T_{x} N=\left\{\left(\varphi_{*} v, \pi_{*} v\right) \mid v \in T_{x} N\right\} .
$$

On the other hand, since $x \in \operatorname{Fix}(\varphi, N)$, we have $\iota_{N} \circ \varphi(x)=\widetilde{\varphi} \circ \iota_{N}(x)=\widetilde{x}$. Therefore, applying (3.2) with $y:=\varphi(x)$, and combining with (3.3), we obtain

$$
T_{\widetilde{x}} \widetilde{N} \cap T_{\widetilde{x}} \widetilde{\varphi}(\widetilde{N})=\left\{\left(\varphi_{*} v, \pi_{*} v\right) \mid v \in T_{x} N, \varphi_{*} v \in T_{\varphi(x)} N, \pi_{*} \varphi_{*} v=\pi_{*} v\right\} .
$$

Claim 1 follows from this.

Assume now that $(N, \varphi)$ is non-degenerate. Let $\widetilde{x}_{0} \in \tilde{N} \cap \widetilde{\varphi}(\tilde{N})$. By assertion (a) there exists $x_{0} \in \operatorname{Fix}(\varphi, N)$ such that $\left(\varphi\left(x_{0}\right), N_{x_{0}}\right)=\widetilde{x}_{0}$. We choose a path $x \in C^{\infty}\left([0,1], N_{x_{0}}\right)$ such that $x(0)=x_{0}$ and $x(1)=\varphi\left(x_{0}\right)$. 
If $0 \neq v \in T_{x_{0}} N \cap T_{x_{0}} \varphi^{-1}(N)$ then by (2.7) and Lemma 3.3 we have $\pi_{*} \varphi_{*} v \neq \pi_{*} v$. Therefore by Claim 1 with $x$ replaced by $x_{0}$ the manifolds $\widetilde{\varphi}(\widetilde{N})$ and $\widetilde{N}$ intersect transversely at $\widetilde{x}_{0}$. It follows that $\widetilde{\varphi}(\widetilde{N}) \pitchfork \widetilde{N}$.

Conversely, assume that $\widetilde{\varphi}(\widetilde{N}) \pitchfork \widetilde{N}$. Let $F \subseteq N$ be a leaf, and $x \in$ $C^{\infty}([0,1], F)$ a path, and assume that $x(1)=\varphi \circ x(0)$, and $0 \neq v \in T_{x(0)} N \cap$ $T_{x(0)} \varphi^{-1}(N)$. By Claim 1 we obtain $\pi_{*} \varphi_{*} v \neq \pi_{*} v$. Therefore, by Lemma 3.3 the inequality $(2.7)$ is satisfied. It follows that $(N, \varphi)$ is non-degenerate. This proves (c) and completes the proof of Lemma 3.2.

Lemma 3.4 (Key Lemma). Let $(M, \omega)$ be a symplectic manifold, $N \subseteq M$ a closed, regular coisotropic submanifold, and let $\widetilde{M}, \widetilde{\omega}$ and $\widetilde{N}$ be defined as in (1.2), (1.3). Then $A(M, \omega, N)=A(\widetilde{M}, \widetilde{\omega}, \widetilde{N})$.

Proof of Lemma 3.4. Let $u \in C^{\infty}(\mathbb{D}, M)$ be a map such that there exists a leaf $F \subseteq N$ satisfying $u\left(S^{1}\right) \subseteq F$. Then the map $\widetilde{u}: \mathbb{D} \rightarrow \widetilde{M}=M \times N_{\omega}$ defined by $\widetilde{u}(z):=\left(u(z), N_{u(1)}\right)$, satisfies $\widetilde{u}\left(S^{1}\right) \subseteq \widetilde{N}$ and $\int_{\mathbb{D}} \widetilde{u}^{*} \widetilde{\omega}=\int_{\mathbb{D}} u^{*} \omega$. It follows that $S(M, \omega, N) \subseteq S(\widetilde{M}, \widetilde{\omega}, \widetilde{N})$.

To show the opposite inclusion, let $\widetilde{u}=\left(v, w^{\prime}\right) \in C^{\infty}(\mathbb{D}, \widetilde{M})$ be a map such that $\widetilde{u}\left(S^{1}\right) \subseteq \widetilde{N}$. We choose a smooth map $\rho:[0,1] \rightarrow[0,1]$ such that

$$
\begin{gathered}
\rho(1 / 2)=1, \quad \rho(r)=r, \forall r \in[0,1 / 4], \quad \rho(r)=1-r, \forall r \in[3 / 4,1], \\
\rho^{\prime}(r)>0, \forall r \in(1 / 4,1 / 2), \quad \rho^{\prime}(r)<0, \forall r \in(3 / 4,1),
\end{gathered}
$$

and all derivatives of $\rho$ vanish at $1 / 2$. We define $\varphi: \mathbb{D} \rightarrow \mathbb{D}$ by $\varphi(r z):=\rho(r) z$, for $r \in[0,1]$ and $z \in S^{1}$.

Claim 1. There exists an extension $u \in C^{\infty}(\mathbb{D}, M)$ of $\left.v \circ \varphi\right|_{\bar{B}_{1 / 2}}$ such that for every $z \in \bar{B}_{1} \backslash B_{1 / 2}$, we have $u(z) \in N$ and $\pi \circ u(z)=w^{\prime} \circ \varphi(z)$.

Proof of Claim 1. We define $f^{\prime}:[0,1] \times S^{1} \rightarrow N_{\omega}$ by $f^{\prime}(t, z):=w^{\prime}(t z)$. For every $z \in S^{1}$ we have by assumption $\widetilde{u}(z) \in \widetilde{N}$, i.e., $\pi \circ v(z)=w^{\prime}(z)=$ $f^{\prime}(1, z)$. Hence $f^{\prime}$ is a smooth homotopy in $N_{\omega}$, ending at the map $\left.\pi \circ v\right|_{S^{1}}$. Since $N$ is closed and the projection $\pi: N \rightarrow N_{\omega}$ is a submersion, results by Ehresmann imply that there exists $f \in C^{\infty}\left([0,1] \times S^{1}, N\right)$ such that $\pi \circ f=f^{\prime}$ and $f(1, \cdot)=\left.v\right|_{S^{1}}$. (See $[\mathbf{E h}]$, the proposition on p. 31 and the second proposition on p. 35.) We define $u: \mathbb{D} \rightarrow M$ by $u(z):=v \circ \varphi(z)$, if $|z| \leq 1 / 2$, and $u(z):=f(\rho(|z|), z /|z|)$, if $1 / 2<|z| \leq 1$. This map has the required properties. This proves Claim 1.

We choose a map $u$ as in Claim 1 . Then $u\left(S^{1}\right)$ is contained in the leaf $w^{\prime}(0)$. Furthermore, since $\varphi$ restricts to an orientation preserving diffeomorphism from $B_{1 / 2}$ onto $B_{1}$, we have $\int_{B_{1 / 2}} u^{*} \omega=\int_{\mathbb{D}} v^{*} \omega$. Furthermore, on $\mathbb{D} \backslash B_{1 / 2}$, we have $u^{*} \omega=u^{*} \pi^{*} \omega_{N}=(\pi \circ u)^{*} \omega_{N}=\varphi^{*} w^{\prime *} \omega_{N}$. Since $\varphi$ restricts to an orientation reversing diffeomorphism from $B_{1} \backslash \bar{B}_{1 / 2}$ onto $B_{1} \backslash\{0\}$, it follows 
that $\int_{\mathbb{D} \backslash B_{1 / 2}} u^{*} \omega=-\int_{\mathbb{D}} w^{\prime *} \omega_{N}$. We obtain $\int_{\mathbb{D}} u^{*} \omega=\int_{\mathbb{D}}\left(v^{*} \omega-w^{\prime *} \omega_{N}\right)=$ $\int_{\mathbb{D}} \widetilde{u}^{*} \widetilde{\omega}$. It follows that $S(\widetilde{M}, \widetilde{\omega}, \widetilde{N}) \subseteq S(M, \omega, N)$. The statement of Lemma 3.4 follows.

\section{Proofs of the main results}

Proof of Theorem 1.1. Let $M, \omega$ and $N$ be as in the hypothesis of this theorem. Without loss of generality we may assume that $N$ is connected. Since $N$ is regular, the symplectic quotient $\left(N_{\omega}, \mathcal{A}_{N, \omega}, \omega_{N}\right)$ of $\left(N,\left.\omega\right|_{N}\right)$ is well defined. We define $\widetilde{M}, \widetilde{\omega}$ and $\widetilde{N}$ as in (1.2), (1.3). Since $N$ is closed, $N_{\omega}$ is closed. By a straightforward argument the product of two bounded symplectic manifolds is bounded, hence $(\widetilde{M}, \widetilde{\omega})=\left(M \times N_{\omega}, \omega \oplus\left(-\omega_{N}\right)\right)$ is bounded. Furthermore, by Lemma $3.2($ b) $\widetilde{N} \subseteq \widetilde{M}$ is a Lagrangian submanifold. It is closed since $N$ is closed. Therefore, we may apply Theorem 3.1 with $M, \omega$ replaced by $\widetilde{M}, \widetilde{\omega}$, and $L:=\widetilde{N}$, to obtain a constant $C$ as in the assertion of that theorem. By Lemma 3.4 this constant has the required property $C \geq A(M, \omega, N)$. Let now $\varphi \in \operatorname{Ham}(M, \omega)$ be such that $(N, \varphi)$ is non-degenerate and $d\left(\varphi, \mathrm{id}_{M}\right)<C$. We define $\widetilde{\varphi}$ as in (3.1). Then $d^{\widetilde{M}, \widetilde{\omega}}\left(\widetilde{\varphi}, \mathrm{id}_{\widetilde{M}}\right) \leq d^{M, \omega}\left(\varphi, \operatorname{id}_{M}\right)<C$, and Lemma 3.2(c) implies that $\widetilde{\varphi}(\widetilde{N}) \pitchfork \widetilde{N}$. Therefore, by the conclusion of Theorem 3.1, we have $|(\widetilde{N} \cap \widetilde{\varphi}(\widetilde{N}))| \geq \sum_{i} b_{i}(\widetilde{N})$. Parts (a) and (b) of Lemma 3.2 imply that $|\operatorname{Fix}(\varphi, N)|=|(\widetilde{N} \cap \widetilde{\varphi}(\widetilde{N}))|$ and $b_{i}(\widetilde{N})=b_{i}(N)$, for every $i=0, \ldots, \operatorname{dim} N$. Inequality (1.1) follows. This proves Theorem 1.1.

Proof of Theorem 1.2.

Claim 1. Assume that the statement of the theorem holds if there exists a closed presymplectic manifold $\left(M^{\prime}, \omega^{\prime}\right)$ and an $\omega^{\prime}$-horizontal distribution $H \subseteq$ $T M^{\prime}$ such that $N$ is the zero-section of $\left(T M^{\prime \omega^{\prime}}\right)^{*}, M$ is an open neighborhood of $N$ and $\omega=\Omega_{\omega^{\prime}, H}$ (see (2.4)). Then it holds in general.

Proof. This follows from Marle's local normal form theorem, see 4.5. Théorème on p. 79 in $[\mathbf{M a}]$.

Let $M, \omega, N, f$ and $\mathcal{U}$ be as in the hypothesis of Theorem 1.2, and assume that there exist $\left(M^{\prime}, \omega^{\prime}\right)$ and $H$ as in Claim 1 . We identify $M^{\prime}$ with $N$, and denote by $\pi:\left(T N^{\omega}\right)^{*} \rightarrow N$ the canonical projection. We choose a Riemannian metric $g$ on $M$. Let $x, y \in M$. For a linear map $T: T_{x} M \rightarrow T_{y} M$ we define $|T|_{\text {op }}:=\max \left\{|T v|\left|v \in T_{x} M:\right| v \mid=1\right\}$, and for a bilinear map $b: T_{x} M \times T_{x} M \rightarrow \mathbb{R}$ we define $|b|:=\max \left\{|b(v, w)|\left|v, w \in T_{x} M,\right| v \mid=\right.$ $|w|=1\}$. We choose $\rho \in C_{\mathrm{c}}^{\infty}(M, \mathbb{R})$ such that $\rho=1$ in a neighborhood of $N$. We define $F:=\rho \cdot(f \circ \pi): M \rightarrow \mathbb{R}$, and denote by $\varphi_{F}^{t}$ the time- $t$-map of $F$, for $t \in \mathbb{R}$. A short argument shows that Crit $f \subseteq \operatorname{Fix}\left(\varphi_{F}^{t}, N\right)$, for $t \in \mathbb{R}$. 
Claim 2. There exists a number $t_{1}>0$ such that for every $t \in\left(0, t_{1}\right]$, we have $\operatorname{Fix}\left(\varphi_{F}^{t}, N\right) \subseteq$ Crit $f$.

Proof of Claim 2. Using Lemma A.6 below, it suffices to prove that there exists a constant $C$ such that for every $t \in[0, \infty)$, and $x_{0} \in \operatorname{Fix}\left(\varphi_{F}^{t}, N\right)$, we have $d\left(x_{0}, \varphi_{F}^{t}\left(x_{0}\right)\right) \leq C\left(\int_{0}^{t}\left|X_{F} \circ \varphi_{F}^{s}\left(x_{0}\right)\right| \mathrm{d} s\right)^{2}$. To see this, we apply Proposition A.4 below with $M, g$ replaced by $N$ and the restriction of $g$ to $N$, and $\mathcal{F}$ the isotropic foliation of $N$. We choose a constant $C^{\prime}:=C$ as in that proposition. Let $t \in[0, \infty)$ and $x_{0} \in \operatorname{Fix}\left(\varphi_{F}^{t}, N\right)$. We define $x:[0, t] \rightarrow N$ by $x(s):=\pi \circ \varphi_{F}^{s}\left(x_{0}\right)$. It follows from an elementary calculation that $\dot{x}(s) \in$ $H_{x(s)}$, for every $s \in[0, t]$. Furthermore, $x(t)=\varphi_{F}^{t}\left(x_{0}\right) \in N_{x(0)}$. Hence the conditions of Proposition A.4 are satisfied. It follows that $d\left(x_{0}, \varphi_{F}^{t}\left(x_{0}\right)\right) \leq$ $C^{\prime}\left(\int_{0}^{t}|\dot{x}| \mathrm{d} s\right)^{2}$. Existence of a constant $C$ as required follows. This proves Claim 2.

We choose $t_{1}$ as in Claim 2. Since $F$ has compact support, there exists $t_{2}>0$ such that $\varphi_{F}^{t} \in \mathcal{U}$, for every $t \in\left[0, t_{2}\right]$.

Claim 3. There exists $t_{3}>0$ such that for every $0<t \leq t_{3}$ the pair $\left(N, \varphi_{F}^{t}\right)$ is non-degenerate.

Proof of Claim 3. Let $x_{0} \in$ Crit $f$ and $t \in \mathbb{R}$. We define

$$
T_{x_{0}}^{t}:=d \varphi_{F}^{t}\left(x_{0}\right)-\mathrm{id}-t d X_{F}\left(x_{0}\right): T_{x_{0}} M \rightarrow T_{x_{0}} M .
$$

We have $T_{x_{0}}^{0}=0$. Since $\left.\frac{\mathrm{d}}{\mathrm{d} t}\right|_{t=0} \mathrm{~d} \varphi_{F}^{t}\left(x_{0}\right)=\mathrm{d} X_{F}\left(x_{0}\right)$, by Taylor's theorem there exists a constant $C_{x_{0}}$ such that $\left|T_{x_{0}}^{t}\right|_{\text {op }} \leq C_{x_{0}} t^{2}$, for every $t \in\left[0, t_{1}\right]$.

A calculation in Darboux charts shows that the bilinear form $B_{x_{0}}$ : $T_{x_{0}} M \times T_{x_{0}} M \ni(v, w) \mapsto \omega\left(\mathrm{d} X_{F}\left(x_{0}\right) v, w\right) \in \mathbb{R}$ is the Hessian of $F$. Since $\left.F\right|_{N}=f$, it follows that the restriction $b_{x_{0}}:=\left.B_{x_{0}}\right|_{T_{x_{0}} N \times T_{x_{0}} N}$ is the Hessian of $f$. We define the linear map $A: T_{x_{0}} N \rightarrow T_{x_{0}} N$ by $g_{x_{0}}(\cdot, A \cdot):=b_{x_{0}}$, and we denote by $V_{+}$and $V_{-}$the direct sum of the positive and negative eigenspaces of $A$, respectively. It follows that $A$ is self-adjoint with respect to $g_{x_{0}}$. Since by assumption $f$ is Morse, the form $b_{x_{0}}$ is nondegenerate, hence $A$ is an isomorphism, and therefore $T_{x_{0}} N=V_{+} \oplus V_{-}$. We define $c_{x_{0}}:=\min \{|\lambda| \mid \lambda$ eigenvalue of $A\}$. Since $f$ is Morse the set Crit $f$ is isolated. Since $N$ is compact, it follows that Crit $f$ is finite. Hence the number $t_{3}:=\min \left(\left\{c_{x_{0}} /\left(2 C_{x_{0}}\left|\omega_{x_{0}}\right|\right) \mid x_{0} \in\right.\right.$ Crit $\left.\left.f\right\} \cup\left\{t_{1}\right\}\right)$ is positive. For $x_{0} \in N$ we denote by $\operatorname{pr}_{x_{0}}: T_{x_{0}} N \rightarrow T_{x_{0}} N / T_{x_{0}} N^{\omega}$ the canonical projection. Let $0<t \leq t_{3}, F \subseteq N$ be a leaf, $x \in C^{\infty}([0,1], F)$ a path and $v \in T_{x(0)} N \cap T_{x(0)}\left(\varphi_{F}^{t}\right)^{-1}(N)$. Assume that $\varphi_{F}^{t}(x(0))=x(1)$, and that

$$
\operatorname{hol}_{x}^{\omega, N} \operatorname{pr}_{x(0)} v=\operatorname{pr}_{x(1)} \mathrm{d} \varphi_{F}^{t}(x(0)) v .
$$

Claim 3 is a consequence of the following. 
Claim 4. We have $v=0$.

Proof of Claim 4. We abbreviate $x_{0}:=x(0)$. Since $x_{0} \in \operatorname{Fix}\left(\varphi_{F}^{t}, N\right)$ and $t \leq t_{1}$, Claim 2 implies that $x_{0} \in$ Crit $f$. We define $A$ and $V_{ \pm}$as above, and $v_{ \pm} \in V_{ \pm}$by $v_{+}+v_{-}:=v$. Since $A$ is $g_{x_{0}}$-self-adjoint, eigenvectors of $A$ for distinct eigenvalues are $g_{x_{0}}$-orthogonal to each other. It follows that $b_{x_{0}}\left(v_{-}, v_{+}\right)=0$, and therefore, $c_{x_{0}}\left|v_{ \pm}\right|^{2} \leq\left|b_{x_{0}}\left(v, v_{ \pm}\right)\right|=\left|\omega\left(\mathrm{d} X_{F}\left(x_{0}\right) v, v_{ \pm}\right)\right|$. Since by assumption $N$ is regular, it follows from (4.2) and Lemmas 3.3 and A.5(b) that $\mathrm{d} \varphi_{F}^{t}\left(x_{0}\right) v-v \in \operatorname{ker} \mathrm{d} \pi_{N}\left(x_{0}\right)=T_{x_{0}} N^{\omega}$. Hence by (4.1) we have $t \omega\left(\mathrm{d} X_{F}\left(x_{0}\right) v, v_{ \pm}\right)=-\omega\left(T_{x_{0}}^{t} v, v_{ \pm}\right)$. Consider the case $\left|v_{+}\right| \geq\left|v_{-}\right|$. Then $|v| \leq \sqrt{2}\left|v_{+}\right|$. It follows that $c_{x_{0}}\left|v_{+}\right|^{2} t \leq \sqrt{2}\left|\omega_{x_{0}}\right|\left|T_{x_{0}}^{t}\right|{ }_{\text {op }}\left|v_{+}\right|^{2}$. Combining this with the inequalities $\left|T_{x_{0}}^{t}\right|_{\text {op }} \leq C_{x_{0}} t^{2}$ and $0<t \leq t_{3}$, it follows that $\left|v_{+}\right|=0$, and therefore $v=v_{+}+v_{-}=0$. The case $\left|v_{-}\right| \geq\left|v_{+}\right|$is treated analogously. This proves Claim 4 and completes the proof of Claim 3.

We choose a number $t_{3}$ as in Claim 3 , and define $\varphi:=\varphi_{F}^{\min \left\{t_{1}, t_{2}, t_{3}\right\}}$. This map has the required properties. This proves Theorem 1.2.

Proof of Proposition 1.3. The first assertion is a consequence of the following two claims. Note that the isotropic leaf through $\Theta \in V(k, n)$ is the orbit $U(k) \cdot \Theta$ of the action of $U(k)$ on $V(k, n)$ by left multiplication.

Claim 1. We have $A\left(\mathbb{C}^{k \times n}, \omega_{0}, V(k, n)\right) \leq \pi$.

Proof of Claim 1. Consider the map $u: \mathbb{D} \rightarrow \mathbb{C}^{k \times n}$ defined by $u_{1}^{1}(z):=z$, for $z \in \mathbb{D}, u_{i}^{i} \equiv 1$, for $i=2, \ldots, k$, and $u^{i}{ }_{j} \equiv 0$, for $i=1, \ldots, k, j=1, \ldots, n$ such that $i \neq j$. Then $u\left(S^{1}\right) \subseteq \mathrm{U}(k) \cdot u(1)$ and $\int_{\mathbb{D}} u^{*} \omega_{0}=\pi$. It follows that $\pi \in S\left(\mathbb{C}^{k \times n}, \omega_{0}, V(k, n)\right)$ (defined as in (2.5)). Claim 1 follows from this.

Claim 2. We have $A\left(\mathbb{C}^{k \times n}, \omega_{0}, V(k, n)\right) \geq \pi$.

Proof of Claim 2. Let $u \in C^{\infty}\left(\mathbb{D}, \mathbb{C}^{k \times n}\right)$, and assume that $\int_{\mathbb{D}} u^{*} \omega_{0}>0$, and that there exists an isotropic leaf $F \subseteq V(k, n)$ such that $u(z) \in F$, for every $z \in S^{1}$. Since the action of $U(k)$ on $V(k, n)$ is free, there exists a unique map $g_{0}: S^{1} \rightarrow U(k)$ such that $u(z)=g_{0}(z) u(1)$, for $z \in S^{1}$. This map is smooth. We define $d$ to be the degree of the map detog $g_{0}: S^{1} \rightarrow S^{1}$. Claim 2 is a consequence of the following.

Claim 3. We have $\int_{\mathbb{D}} u^{*} \omega_{0}=\mathrm{d} \pi$.

Proof of Claim 3. For $a_{1}, \ldots, a_{k} \in \mathbb{C}$ we denote by $\operatorname{diag}\left(a_{1}, \ldots, a_{k}\right) \in \mathbb{C}^{k \times k}$ the diagonal matrix with entries $a_{1}, \ldots, a_{k}$. We define $h_{0}: S^{1} \rightarrow U(k)$ by $h_{0}(z):=\operatorname{diag}\left(z^{d}, 1, \ldots, 1\right) g_{0}(z)^{-1}$. The map det $\circ h_{0}: S^{1} \rightarrow S^{1}$ has degree 0 . Since the determinant induces an isomorphism of the fundamental groups of $U(k)$ and $S^{1}$, it follows that there exists a continuous map $h: \mathbb{D} \rightarrow$ $U(k)$ such that $\left.h\right|_{S^{1}}=h_{0}$. We may assume without loss of generality that $h$ is smooth. Let $\mu: \mathbb{C}^{k \times n} \rightarrow \operatorname{Lie} \mathrm{U}(k)$ be a moment map for the action 
of $U(k)$ on $V(k, n)$. By a straightforward calculation, we have $(h u)^{*} \omega_{0}=$ $u^{*} \omega_{0}-d\left\langle\mu \circ u, h^{-1} d h\right\rangle$, see Lemma 9 in [Zi1]. By Stokes' theorem, it follows that $\int u^{*} \omega_{0}=\int_{\mathbb{D}}(h u)^{*} \omega_{0}$. We define the map $v: \mathbb{D} \rightarrow \mathbb{C}^{k \times n}$ by $v(z):=$ $\operatorname{diag}\left(z^{d}, 1, \ldots, 1\right) u(1)$, for $z \in \mathbb{D}$. Then $(h u)(z)=\left(h_{0} u\right)(z)=v(z)$, for $z \in S^{1}$. Therefore, $\int_{\mathbb{D}}(h u)^{*} \omega_{0}=\int_{\mathbb{D}} v^{*} \omega_{0}=\mathrm{d} \pi \sum_{j=1, \ldots, n}\left|u^{1}{ }_{j}(1)\right|^{2}=\mathrm{d} \pi$. This proves Claim 3 and hence Claim 2.

To prove the second assertion, let $C>\pi$. There exists $\widetilde{H} \in C_{\mathrm{c}}^{\infty}(\mathbb{C}, \mathbb{R})$ such that $\varphi_{\widetilde{H}}^{1}(\mathbb{D}) \cap \mathbb{D}=\emptyset$, and $\|\widetilde{H}\|:=\int_{0}^{1}\left(\max _{\mathbb{C}^{k \times n}} \widetilde{H}(t, \cdot)-\min _{\mathbb{C}^{k \times n}} \widetilde{H}(t, \cdot)\right) \mathrm{d} t<$ $C$. (See for example the proof of Proposition 1.4. in [Ho].) We define $\pi$ : $\mathbb{C}^{k \times n} \rightarrow \mathbb{C}$ by $\pi(\Theta):=\Theta^{1}{ }_{1}$. We choose $\rho \in C_{\mathrm{c}}^{\infty}\left(\mathbb{C}^{k \times n},[0,1]\right)$, such that $\rho=1$ on $\bigcup_{t \in[0,1]} \varphi_{\widetilde{H} \circ \pi}^{t}(V(k, n))$. Furthermore, we define $H:=\rho \cdot(\widetilde{H} \circ \pi): \mathbb{C}^{k \times n} \rightarrow$ $\mathbb{R}$, and $\varphi:=\varphi_{H}^{1}$. Then $\varphi \in \operatorname{Ham}_{\mathrm{c}}\left(\mathbb{C}^{k \times n}, \omega_{0}\right)$, and $d_{\mathrm{c}}(\varphi, \mathrm{id}) \leq\|H\|=\|\widetilde{H}\|<$ $C$. The facts $\pi(V(k, n))=\mathbb{D}, \pi \circ \varphi_{\widetilde{H} \circ \pi}^{1}=\varphi_{\widetilde{H}}^{1} \circ \pi$ and $\varphi_{\widetilde{H}}^{1}(\mathbb{D}) \cap \mathbb{D}=\emptyset$ imply that $\emptyset=V(k, n) \cap \varphi_{\widetilde{H} \circ \pi}^{1}(V(k, n))=V(k, n) \cap \varphi_{H}^{1}(V(k, n))$. Hence $\varphi$ has the required properties. This proves Proposition 1.3.

Proof of Proposition 1.4. Let $M_{i}, \omega_{i}, L, M, \omega, N$ and $\psi$ be as in the hypothesis of the Conjecture. Without loss of generality we may assume that $M_{1}$ and $L$ are connected. We define

$$
\begin{gathered}
\widetilde{M}:=M_{1} \times M_{2} \times M_{1}, \quad \widetilde{\omega}:=\omega_{1} \oplus \omega_{2} \oplus\left(-\omega_{1}\right), \\
\widetilde{N}:=\left\{\left(x_{1}, x_{2}, x_{1}\right) \in M \mid x_{1} \in M_{1}, x_{2} \in L\right\}, \quad \widetilde{\varphi}:=\varphi \times \operatorname{id}_{M_{1}} .
\end{gathered}
$$

Then $\widetilde{N}$ is a closed Lagrangian submanifold of $\widetilde{M}$. The map $\widetilde{\psi}: \widetilde{M} \rightarrow \widetilde{M}$ defined by $\widetilde{\psi}\left(x_{1}, x_{2}, y\right):=\left(y, \psi\left(x_{2}\right), x_{1}\right)$ is an $\widetilde{\omega}$-anti-symplectic involution whose fixed point set equals $\widetilde{N}$. Furthermore, $N=M_{1} \times L \subseteq M=$ $M_{1} \times M_{2}$ is a regular $\omega$-coisotropic submanifold, and the symplectic quotient $\left(N_{\omega}, \mathcal{A}_{N, \omega}, \omega_{N}\right)$ of $\left(N,\left.\omega\right|_{N}\right)$ is isomorphic to $\left(M_{1}, \omega_{1}\right)$ via the map $M_{1} \ni y \mapsto\{y\} \times L \in N_{\omega}$. Via this map, the definitions of $\widetilde{M}, \widetilde{\omega}$ and $\widetilde{\varphi}$ agree with $(1.2),(1.3),(3.1)$. Hence by Lemma $3.2(\mathrm{c})$, we have $\widetilde{N} \pitchfork \widetilde{\varphi}(\widetilde{N})$. Thus $\widetilde{M}, \widetilde{\omega}$ and $\widetilde{N}$ satisfy the hypotheses of the Lagrangian AGC. Supposing that this conjecture is true, it follows that $|\widetilde{N} \cap \widetilde{\varphi}(\widetilde{N})| \geq \sum_{i} b_{i}(\widetilde{N})$. Inequality (1.1) follows now from Lemma 3.2(a). This proves Proposition 1.4.

Proof of Corollary 1.5. Let $(M, \omega)$ be a bounded and aspherical symplectic manifold, and $\left(M^{\prime}, \omega^{\prime}\right)$ a closed and regular presymplectic manifold of corank $\operatorname{dim} M-\operatorname{dim} M^{\prime}$. Assume that $M^{\prime}$ has a simply connected isotropic leaf $F_{0}$, and that there exists an embedding $\psi: M^{\prime} \rightarrow M$ satisfying $\psi^{*} \omega=\omega^{\prime}$. Let $\varphi \in \operatorname{Ham}(M, \omega)$. It suffices to prove that $N:=\psi\left(M^{\prime}\right)$ intersects $\varphi(N)$. Replacing $M^{\prime}$ by the connected component of $M^{\prime}$ containing $F_{0}$, we may assume without loss of generality that $M^{\prime}$ is connected. It follows from Proposition A.1 below that the submanifold $\psi\left(M^{\prime}\right) \subseteq M$ is coisotropic. 
We choose a constant $C>0$ as in Theorem 1.1. If $(N, \varphi)$ is degenerate, then by definition $\operatorname{Fix}(\varphi, N) \neq \emptyset$, and hence $N \cap \varphi(N) \neq \emptyset$. So assume that $(N, \varphi)$ is non-degenerate.

Claim 1. We have $A(M, \omega, N)=\infty$.

Proof of Claim 1. Let $u \in C^{\infty}(\mathbb{D}, M)$ be a smooth map such that there exists $F \in N_{\omega}$ such that $u\left(S^{1}\right) \subseteq F$. Since $N$ is closed and the projection $\pi_{N}: N \rightarrow N_{\omega}$ is a submersion, by the proposition on p. 31 in $[\mathbf{E h}]$ it is a smooth fiber bundle. Since $N$ is connected, it follows that $F$ is diffeomorphic to $F_{0}$, and therefore simply connected. Hence there exists a smooth map $v: \mathbb{D} \rightarrow F$ such that $u$ and $v$ agree on the boundary $S^{1}$. We choose a smooth map $\rho:[0,1] \rightarrow[0,1]$ such that $\rho(r)=r$ for $r \leq 1 / 2, \rho(1)=1$, $\rho^{\prime}(r)>0$, for every $r \in(0,1)$, and all derivatives of $\rho$ vanish at $r=1$. We define $f: \mathbb{D} \rightarrow \mathbb{D}$ by $f(r z):=\rho(r) z$, for $r \in[0,1]$ and $z \in S^{1}$. We denote by $\overline{\mathbb{D}}$ the disk with the reversed orientation, and by $w: S^{2} \cong \mathbb{D} \# \overline{\mathbb{D}} \rightarrow M$ the concatenation of $u \circ f$ and $v \circ f$. This map is smooth, and since $(M, \omega)$ is aspherical, we have $0=\int_{S^{2}} w^{*} \omega=\int_{\mathbb{D}} f^{*} u^{*} \omega-\int_{\mathbb{D}} f^{*} v^{*} \omega$. Since $v(\mathbb{D}) \subseteq F$, we have $f^{*} v^{*} \omega=0$. It follows that $\int_{\mathbb{D}} u^{*} \omega=\int_{B_{1}} f^{*} u^{*} \omega=0$. Claim 1 follows from this.

Claim 1 implies that $C=\infty$, and hence $d(\varphi$, id $)<C$. Therefore, the conditions of Theorem 1.1 are satisfied. Applying this theorem, inequality (1.1) follows, and therefore $N \cap \varphi(N) \neq \emptyset$. This proves Corollary 1.5.

\section{Appendix A. Auxiliary results}

The following result was used in Section 1 and in the proof of Corollary 1.5, and it will be used to prove Proposition A.2 below. Its proof involves only linear algebra and is therefore omitted.

Proposition A.1. Let $(M, \omega)$ be a presymplectic manifold, $M^{\prime}$ a manifold, $\psi: M^{\prime} \rightarrow M$ an immersion and $\omega^{\prime}:=\psi^{*} \omega$. If $\omega^{\prime}$ has constant corank then

$$
\operatorname{dim} M^{\prime}+\operatorname{corank} \omega^{\prime} \leq \operatorname{dim} M+\operatorname{corank} \omega .
$$

Suppose now that $\psi$ is an embedding. Then $\psi\left(M^{\prime}\right) \subseteq M$ is coisotropic if and only if $\omega^{\prime}$ has constant corank and equality in (A.1) holds.

The next result was used in Section 1 . Let $(X, \sigma)$ be a closed symplectic manifold, $\pi: E \rightarrow X$ a closed smooth fiber bundle, $H \subseteq T E$ a horizontal subbundle and let $N, \pi_{X}, \iota_{H}, \omega:=\Omega_{\sigma, H}$ and $M$ be as in the construction explained in that section.

Proposition A.2. $N \subseteq M$ is a regular coisotropic submanifold. Furthermore, if $(X, \sigma)$ is aspherical then $A(M, \omega, N)=\infty$.

For the proof of Proposition A.2 we need the following. We denote by $i_{E}$ the embedding of $E$ as the zero section $N \subseteq V^{*} E$. 
Lemma A.3. We have $\pi^{*} \sigma=i_{E}^{*} \omega$.

Proof of Lemma A.3. We denote by $j_{E}$ the embedding of $E$ as the zerosection of $T^{*} E$. Then $\iota_{H} \circ i_{E}=j_{E}$, and therefore, denoting by $\lambda_{\text {can }}$ the canonical one-form on $T^{*} E$, we obtain $i_{E}^{*} \iota_{H}^{*} \omega_{\text {can }}=-d j_{E}^{*} \lambda_{\text {can }}=-d 0=0$. Since $\pi_{X} \circ i_{E}=\pi$, it follows that $\pi^{*} \sigma=i_{E}^{*}\left(\pi_{X}^{*} \sigma+\iota_{H}^{*} \omega_{\text {can }}\right)=i_{E}^{*} \omega$. This proves Lemma A.3.

Proof of Proposition A.2. If follows from Lemma A.3 and Proposition A.1 that $N \subseteq M$ is a coisotropic submanifold. Regularity follows from an elementary argument. Assume now that $(X, \sigma)$ is aspherical. To prove that $A(M, \omega, N)=\infty$, let $u \in C^{\infty}(\mathbb{D}, M)$ be a map such that there exists a leaf $F \in N_{\omega}$ satisfying $u\left(S^{1}\right) \subseteq F$. It suffices to prove the following.

Claim 1. We have $\int_{\mathbb{D}} u^{*} \omega=0$.

Proof of Claim 1. We denote by $\pi_{0}$ the canonical projection from $V^{*} E$ to its zero-section $N$. We choose a smooth function $\rho:[0,1] \rightarrow[0,1]$ such that $\rho(r)=r$, for $r \leq 1 / 3$, and $\rho(r)=1$, for $r \geq 2 / 3$. We define $u_{0}: \mathbb{D} \rightarrow M$ by $u_{0}(r z):=\pi_{0} \circ u(\rho(r) z)$, for $r \in[0,1]$ and $z \in S^{1}$. It follows from an elementary argument involving Stokes' Theorem that $\int_{\mathbb{D}} u^{*} \omega=\int_{\mathbb{D}} u_{0}^{*} \omega$. We claim that $\int_{\mathbb{D}} u_{0}^{*} \omega=0$. To see this, we choose an orientation preserving diffeomorphism $\varphi: \mathbb{C} \rightarrow B_{1}$, and we define the map $f: S^{2} \cong \mathbb{C} \cup\{\infty\} \rightarrow X$ by $f(z):=\pi_{X} \circ u_{0} \circ \varphi(z)$, if $z \in \mathbb{C}$, and $f(z):=\pi_{X} \circ u(1)$, if $z=\infty$. By an elementary argument this map is smooth. Therefore, by symplectic asphericity of $X$ we have $0=\int_{S^{2}} f^{*} \sigma=\int_{\mathbb{C}}\left(\pi_{X} \circ u_{0} \circ \varphi\right)^{*} \sigma=\int_{B_{1}}\left(\pi_{X} \circ u_{0}\right)^{*} \sigma$. Let $v_{0}: \mathbb{D} \rightarrow E$ be the unique map such that $i_{E} \circ v_{0}=u_{0}$. Then $\pi_{X} \circ u_{0}=$ $\pi \circ v_{0}$, and hence using Lemma A.3, $\left(\pi_{X} \circ u_{0}\right)^{*} \sigma=v_{0}^{*} \pi^{*} \sigma=v_{0}^{*} i_{E}^{*} \omega=u_{0}^{*} \omega$. It follows that $\int_{\mathbb{D}} u_{0}^{*} \omega=0$. This completes the proof of Claim 1 and hence of Proposition A.2.

Proof of Proposition 2.1. Let $M, \mathcal{F}, F, a, b, x, N$ and $y_{0}$ be as in the hypothesis. To prove statement (a), let $T: T_{y_{0}} N \rightarrow T_{x(a)} M$ be a linear map. We denote by $\pi_{2}:[a, b] \times M \rightarrow M$ the projection onto the second factor. By elementary arguments there exist $f \in C^{\infty}(N, M)$ such that $f\left(y_{0}\right)=x(a)$ and $d f\left(y_{0}\right)=T$, and a smooth section $s:[a, b] \times M \rightarrow \pi_{2}^{*} T \mathcal{F}$ of compact support, such that $s(t, x(t))=\dot{x}(t)$, for every $t \in[a, b]$. We define $u:[a, b] \times N \rightarrow M$ to be the unique solution of the equations

$$
\partial_{t} u(t, y)=s(t, y), \quad u(a, y)=f(y), \quad \forall t \in[a, b], y \in N .
$$

This map has the required properties. This proves (a).

To prove statement (b), let $u$ and $u^{\prime}$ be as in the hypothesis. Consider

$$
S:=\left\{t \in[a, b] \mid \operatorname{pr}^{\mathcal{F}} d(u(t, \cdot))\left(y_{0}\right)=\operatorname{pr}^{\mathcal{F}} d\left(u^{\prime}(t, \cdot)\right)\left(y_{0}\right)\right\} .
$$


By (2.3) this set contains $a$. Furthermore, it is a closed subset of $[a, b]$. It follows from an elementary argument that it is open, and therefore $S=[a, b]$. This proves (b) and completes the proof of Proposition 2.1.

The next result was used in the proof of Theorem 1.2.

Proposition A.4. Let $(M, g)$ be a closed Riemannian manifold, $\mathcal{F}$ a regular foliation on $M$ and $H \subseteq T M$ an $\mathcal{F}$-horizontal distribution. Then there exists a constant $C$ such that for every $t \geq 0$ and every $x \in C^{\infty}([0, t], M)$ the following holds. If $\dot{x}(s) \in H_{x(s)}$, for every $s \in[0, t]$, and $x(t) \in \mathcal{F}_{x(0)}$, then $d(x(0), x(t)) \leq C \ell(x)^{2}$.

Proof of Proposition A.4. We denote by $n$ and $k$ the dimension of $M$ and of the leaves of $\mathcal{F}$ respectively. Since $\mathcal{F}$ is regular, there exists a unique smooth structure on the set of leaves $M / R^{\mathcal{F}}$ such that the canonical projection $\pi^{\mathcal{F}}$ : $M \rightarrow M / R^{\mathcal{F}}$ is a submersion, see Lemma A.5 below. Hence by Ehresmann's Theorem (proposition on p. 31 in $[\mathbf{E h}]), \pi^{\mathcal{F}}$ is a smooth fiber bundle. It follows that there exists a finite atlas $\mathcal{A}$ of surjective foliation charts $\varphi$ : $U \rightarrow \mathbb{R}^{n} \cong \mathbb{R}^{n-k} \times \mathbb{R}^{k}$, such that $\bigcup_{(U, \varphi) \in \mathcal{A}} \varphi^{-1}\left(B_{1}\right)=M$, and for every $(U, \varphi) \in \mathcal{A}$ and $x \in U$ the set $\varphi\left(\mathcal{F}_{x} \cap U\right)$ is connected. For $(U, \varphi) \in \mathcal{A}$ we define $\varepsilon_{\varphi}$ to be the distance (with respect to $g$ ) between $\varphi^{-1}\left(\bar{B}_{1}\right)$ and $M \backslash \varphi^{-1}\left(B_{2}\right)$, and we set $\varepsilon:=\min \left\{\varepsilon_{\varphi} \mid(U, \varphi) \in \mathcal{A}\right\}$. Proposition A.4 is a consequence of the following.

Claim 1. There exists a constant $C$ such that for every $t \in[0, \infty)$ and every $x \in C^{\infty}([0, t], M)$ the following holds. If $\dot{x}\left(t^{\prime}\right) \in H_{x\left(t^{\prime}\right)}$, for every $t^{\prime} \in[0, t]$, $x(t) \in \mathcal{F}_{x(0)}$, and $\ell(x) \leq \varepsilon$ then $d(x(0), x(t)) \leq C \ell(x)^{2}$.

Proof of Claim 1. Let $x \in C^{\infty}([0, t], M)$ be such that $\dot{x}\left(t^{\prime}\right) \in H_{x\left(t^{\prime}\right)}, x(t) \in$ $\mathcal{F}_{x(0)}$, and $\ell(x) \leq \varepsilon$. We choose a chart $(U, \varphi) \in \mathcal{A}$ such that $x(0) \in \varphi^{-1}\left(B_{1}\right)$. By the choice of $\varepsilon$ it follows that $x\left(t^{\prime}\right) \in \varphi^{-1}\left(B_{2}\right)$, for every $t^{\prime} \in[0, t]$. Hence we may define $(a, b):=\varphi \circ x:[0, t] \rightarrow \mathbb{R}^{n}=\mathbb{R}^{n-k} \times \mathbb{R}^{k}$. By the choice of $\mathcal{A}$, the set $\varphi\left(\mathcal{F}_{x(0)} \cap U\right)$ is connected, hence it equals $\{a(0)\} \times \mathbb{R}^{k}$. Since $x(t) \in$ $\mathcal{F}_{x(0)}$, it follows that $a(t)=a(0)$. Therefore, denoting by $|\cdot|_{0}$ the standard norm on Euclidian space, we have $|(a, b)(t)-(a, b)(0)|_{0}=|b(t)-b(0)|_{0}$. We define the map $(u, v):[0,1] \times[0, t] \rightarrow \mathbb{R}^{n-k} \times \mathbb{R}^{k}$ by

$$
(u, v)\left(s, t^{\prime}\right):=(a, b)(0)+s\left((a, b)\left(t^{\prime}\right)-(a, b)(0)\right) .
$$

Furthermore, we define $\alpha: \mathbb{R}^{n} \rightarrow \mathbb{R}^{k \times(n-k)}$ as follows. Namely, for $x \in$ $\mathbb{R}^{n}$ we define $\alpha(x)$ to be the unique real $k \times(n-k)$ matrix satisfying $\left\{(w, \alpha(x) w) \mid w \in \mathbb{R}^{n-k}\right\}=d \varphi H_{\varphi^{-1}(x)}$. Since $H$ is horizontal, $\alpha$ is well defined. For $t^{\prime} \in[0, t]$ we have $\dot{b}\left(t^{\prime}\right)=\int_{0}^{1} \partial_{s}\left(\alpha \circ(u, v)\left(s, t^{\prime}\right) \partial_{t} u\left(s, t^{\prime}\right)\right) \mathrm{d} s$. Therefore, a short calculation using $a(t)=a(0)$, implies that $b(t)-b(0)=$ $\int_{0}^{t} \int_{0}^{1}\left(\left(d \alpha \partial_{s}(u, v)\right) \partial_{t} u-\left(d \alpha \partial_{t}(u, v)\right) \partial_{s} u\right) \mathrm{d} s \mathrm{~d} t^{\prime}$. Claim 1 is a consequence of this. This proves Proposition A.4. 
Lemma A.5. Let $M$ be a set with a smooth structure and $R$ an equivalence relation on $M$. Then the following holds:

(a) There is at most one smooth structure on $M^{\prime}:=M / R$ such that the quotient map $\pi: M \rightarrow M^{\prime}$ is a submersion.

Assume that $R$ is the leaf relation of some foliation $\mathcal{F}$ on $M$. Then:

(b) If there is a smooth structure on $M^{\prime}$ as in (a) then $\operatorname{ker} \mathrm{d} \pi(x)=T_{x} \mathcal{F}$, for every $x \in M$.

(c) $R$ is a closed subset of $M \times M$ if and only if $M^{\prime}$ (equipped with the quotient topology) is Hausdorff.

Assume that $R$ is the leaf relation of some foliation $\mathcal{F}$ and the topology on $M$ is Hausdorff and second countable. Then:

(d) The quotient topology on $M^{\prime}$ is second countable.

(e) The following conditions are equivalent:

(i) There exists a smooth structure on $M^{\prime}$ as in (a).

(ii) $R$ is a submanifold of $M \times M$.

(f) Assume that there is a smooth structure $\mathcal{A}$ on $M^{\prime}$ as in (a). Let $F$ be a leaf of $\mathcal{F}, x \in C^{\infty}([0,1], F)$, and $v_{i} \in T_{x(i)} M$, for $i=0,1$, be such that $\operatorname{pr}^{\mathcal{F}} v_{1}=\operatorname{hol}_{x}^{\mathcal{F}} \operatorname{pr}^{\mathcal{F}} v_{0}$. Then $\mathrm{d} \pi(x(0)) v_{0}=\mathrm{d} \pi(x(1)) v_{1}$, where the differentials are defined with respect to $\mathcal{A}$.

Proof of Lemma A.5. Statements (a,b,c,d) and (f) follow from elementary arguments. To prove assertion (e), assume that $R$ is the leaf relation of some foliation $\mathcal{F}$ and $M$ is Hausdorff and second countable. It follows from Proposition 2.1(a) that the projection onto the first factor $\pi_{1}: R \rightarrow M$ is a submersion. Assertion (e) is a consequence of this and a theorem by Godement (see Theorem 3.5.25 in the book [AMR]). This concludes the proof of Lemma A.5.

The next lemma was used in the proof of Theorem 1.2. Let $M$ be a $C^{1}$ manifold, and $X$ a complete $C^{1}$-vector field on $M$. We denote by $\varphi_{X}^{t}$ the time-t-map generated by $X$, for $t \in \mathbb{R}$. For a pair $\left(t, x_{0}\right) \in[0, \infty) \times M$ we write $\ell\left(t, x_{0}\right):=\ell\left([0, t] \ni s \mapsto \varphi_{X}^{s}\left(x_{0}\right) \in M\right)$.

Lemma A.6 (Fast almost periodic orbits). Let $(M, g)$ be a Riemannian $C^{2}$-manifold, $X$ a $C^{1}$-vector field on $M$ with compact support, and $f:[0, \infty) \rightarrow[0, \infty)$ a continuous function such that $f(0)=0$. Then there exists a constant $\varepsilon>0$ such that for every $\left(t, x_{0}\right) \in[0, \varepsilon] \times M$ satisfying $d\left(x_{0}, \varphi_{X}^{t}\left(x_{0}\right)\right) \leq \ell\left(t, x_{0}\right) f\left(\ell\left(t, x_{0}\right)\right)$, we have $X\left(x_{0}\right)=0$.

The proof of this lemma is based on an idea from the proof of Proposition 17, p. 184 in the book $[\mathbf{H Z}]$. We need the following.

Remark A.7. If $t \geq 0$ and $x \in W^{1,1}([0, t], \mathbb{R})$ satisfy $\int_{0}^{t} x(s) \mathrm{d} s=0$ then $\|x\|_{L^{1}([0, t])} \leq t\|\dot{x}\|_{L^{1}([0, t])}$. To see this, we choose a point $t_{0} \in[0, t]$ such that $x\left(t_{0}\right)=0$, and observe $\left|x\left(t_{1}\right)\right|=\left|\int_{t_{0}}^{t_{1}} \dot{x}(s) \mathrm{d} s\right| \leq \int_{0}^{t}|\dot{x}(s)| \mathrm{d} s$, for $t_{1} \in[0, t]$. 
Proof of Lemma A.6. Let $M, g, X$ and $f$ be as in the hypothesis. We denote by $K \subseteq M$ the support of $X$, and by $n$ the dimension of $M$. We choose a finite set $\mathcal{A}$ of surjective $C^{2}$-charts $\varphi: U \subseteq M \rightarrow \mathbb{R}^{n}$, such that $K \subseteq \bigcup_{(U, \varphi) \in \mathcal{A}} \varphi^{-1}\left(B_{1}\right)$. For $(U, \varphi) \in \mathcal{A}$ we define $\varepsilon_{\varphi}$ to be the distance between $\varphi^{-1}\left(\bar{B}_{1}\right)$ and $M \backslash \varphi^{-1}\left(B_{2}\right)$, and we set $\varepsilon_{1}:=$ $\min \left\{\varepsilon_{\varphi} / \max _{K}|X| \mid(U, \varphi) \in \mathcal{A}\right\}$. For $v \in \mathbb{R}^{n}$ we denote $|v|_{1}:=\sum_{i=1}^{n}\left|v^{i}\right|$. Let $x_{0} \in M,(U, \varphi) \in \mathcal{A}$ be a chart such that $x_{0} \in \varphi^{-1}\left(B_{1}\right)$, and $s \in\left[0, \varepsilon_{1}\right]$. Then $\varphi_{X}^{s}\left(x_{0}\right) \in \varphi^{-1}\left(\bar{B}_{2}\right)$, hence the point $\varphi \circ \varphi_{X}^{s}\left(x_{0}\right) \in \mathbb{R}^{n}$ is well defined.

Claim 1. There exists $C \geq 0$ such that for every $(U, \varphi) \in \mathcal{A}, x_{0} \in \varphi^{-1}\left(B_{1}\right)$ and $t \in\left[0, \varepsilon_{1}\right]$ satisfying $d\left(x_{0}, \varphi_{X}^{t}\left(x_{0}\right)\right) \leq \ell\left(t, x_{0}\right) f\left(\ell\left(t, x_{0}\right)\right)$, the inequality $\left(C t+C f\left(\ell\left(t, x_{0}\right)\right)-1\right) \int_{0}^{t}\left|\frac{\mathrm{d}}{\mathrm{d} s}\left(\varphi \circ \varphi_{X}^{s}\left(x_{0}\right)\right)\right|_{1} \mathrm{~d} s \geq 0$ holds.

Proof of Claim 1. Let $(U, \varphi), x_{0}$ and $t$ be as in the hypothesis. We define $y:[0, t] \rightarrow \mathbb{R}^{n}$ by $y(s):=\varphi \circ \varphi_{X}^{s}\left(x_{0}\right), v:=y(0)-y(t), z:[0,1] \rightarrow \mathbb{R}^{n}$ by $z(s):=\dot{y}(s)+v / t$, and $C_{0}:=\max \left\{\left|d\left(\varphi_{*} X\right)(x) w\right|_{1} \mid x \in \bar{B}_{2}, w \in \mathbb{R}^{n}\right.$ : $\left.|w|_{1}=1\right\}$. It follows from Remark A.7 that $\|z\|_{1} \leq t\|\dot{z}\|_{1} \leq C_{0} t\|\dot{y}\|_{1}$, where the $L^{1}$-norms are taken with respect to $|\cdot|_{1}$. Since $\|\dot{y}\|_{1} \leq\|z\|_{1}+|v|_{1}$, it follows that $\|\dot{y}\|_{1} \leq C_{0} t\|\dot{y}\|_{1}+|v|_{1}$. Claim 1 is a consequence of this and the inequality $d\left(x_{0}, \varphi_{X}^{t}\left(x_{0}\right)\right) \leq \ell\left(t, x_{0}\right) f\left(\ell\left(t, x_{0}\right)\right)$.

We choose $C \geq 0$ as in Claim 1 , and $0<\varepsilon \leq \varepsilon_{1}$ so small that $C \varepsilon+C \max \left\{f(a)\left|0 \leq a \leq \varepsilon \max _{K}\right| X \mid\right\}<1$. Let $\left(t, x_{0}\right)$ be as in the hypothesis of Lemma A.6. It follows from the statement of Claim 1 that $\frac{\mathrm{d}}{\mathrm{d} s}\left(\varphi \circ \varphi_{X}^{s}\left(x_{0}\right)\right)=0$, for every $s \in[0, t]$, and therefore $X\left(x_{0}\right)=0$. This proves Lemma A.6.

\section{References}

[AMR] R. Abraham, J. E. Marsden and T. Ratiu, Manifolds, tensor analysis, and applications, 2nd ed., Applied Mathematical Sciences, 75, Springer, Berlin, 1988.

[AF] P. Albers and U. Frauenfelder, Leaf-wise intersections and Rabinowitz Floer homology, preprint, October 2008, arXiv:0810.3845v1

[An] D. Andrica, Critical point theory and some applications, Cluj University Press, Cluj-Napoca, Romania, 2005.

[Ar] V. I. Arnol'd, Sur une propriété topologique des applications globalement canoniques de la mécanique classique, C. R. Acad. Sci. Paris 261 (1965), 3719-3722.

[ACL] M. Audin, A. Cannas da Silva and E. Lerman, Symplectic geometry of integrable Hamiltonian systems, Advanced Courses in Mathematics, CRM Barcelona, Birkhäuser, 2003.

[ALP] M. Audin, F. Lalonde and L. Polterovich, Symplectic rigidity: Lagrangian submanifolds, in Holomorphic curves in symplectic geometry, Progr. Math. 117, (H. Bass, J. Oesterlé, and A. Weinstein eds.), Birkhäuser, Basel, 1994, 271-321. 
[Ba] A. Banyaga, On fixed points of symplectic maps, Invent. Math. 56 (3) (1980), $215-229$

[CC] A. Candel and L. Conlon, Foliations II, Graduate Studies in Mathematics, 60, AMS, Providence, RI, 2003.

[Ch] Yu. V. Chekanov, Lagrangian intersections, symplectic energy, and areas of holomorphic curves, Duke Math. J. 95 (1) (1998), 213-226.

[Dr] D. Dragnev, Symplectic rigidity, symplectic fixed points, and global perturbations of Hamiltonian systems, Comm. Pure Appl. Math. 61 (3) (2008), 346-370.

[DV] I. A. Dynnikov and A. P. Veselov, Integrable Gradient Flows and Morse Theory, Algebra i Analiz 8 (3) (1996), 78-103, translation in St. Petersburg Math. J. 8 (3) (1997), 429-446

[Eh] C. Ehresmann, Les connexions infinitésimales dans un espace fibré différentiable, Colloque de topologie (espaces fibrés), Bruxelles, 1950, 29-55.

[EH] I. Ekeland and H. Hofer, Two symplectic fixed-point theorems with applications to Hamiltonian dynamics, J. Math. Pures Appl. (9) 68 (4) (1989), 467-489 (1990).

[Fl] A. Floer, Morse theory for Lagrangian intersections, J. Diff. Geom. 28 (3) (1988), 513-547.

[Fr] U. Frauenfelder, The Arnold-Givental conjecture and moment floer homology, Int. Math. Res. Not. 42 (2004), 2179-2269.

[Gin] V. Ginzburg, Coisotropic intersections, Duke Math. J. 140 (1) (2007), 111-163.

[GHV] W. Greub, S. Halperin and R. Vanstone, Connections, curvature, and cohomology, Vol. II: Lie groups, principal bundles, and characteristic classes, Pure Appl. Math. 47-II, 1973, xxi + 541 pp.

[GGK] V. Guillemin, V. Ginzburg and Y. Karshon, Moment maps, cobordisms, and Hamiltonian group actions, Mathematical Surveys and Monographs, 98, AMS, Providence, RI, 2002.

[Gü] B. Gürel, Leaf-wise coisotropic intersections, preprint, May 2009, arXiv:0905.4139v1, accepted by Int. Math. Res. Not.

[Ho] H. Hofer, On the topological properties of symplectic maps, Proc. Roy. Soc. Edinburgh Sect. A 115 (1-2) (1990), 25-38.

[HZ] H. Hofer, E. Zehnder, Symplectic invariants and Hamiltonian dynamics, Birkhäuser Advanced Texts, Basel Textbooks, 1994.

[Ke] E. Kerman, Displacement energy of coisotropic submanifolds and Hofer's geometry, J. Mod. Dyn. 2 (3) (2008), 471-497.

[Ma] C.-M. Marle, Sous-variétés de rang constant d'une variété symplectique, Astérisque, Soc. Math. France, Paris, 1983, 107-108.

[MS] D. McDuff and D.A. Salamon, Introduction to symplectic topology, 2nd ed., Oxford Mathematical Monographs, The Clarendon Press, Oxford University Press, New York, 1998.

[MM] I. Moerdijk and J. Mrčun, Introduction to foliations and Lie groupoids, Cambridge Studies in Advanced Mathematics, 91, Cambridge University Press, Cambridge, 2003.

[Mos] J. Moser, A fixed point theorem in symplectic geometry, Acta Math. 141 (1-2) (1978), 17-34. 
[TT] C.-L. Terng and G. Thorbergsson, Taut immersions into complete Riemannian manifolds, in Tight and taut submanifolds, Math. Sci. Res. Inst. Publ., 32, Cambridge University Press, Cambridge, 1997, 181-228.

[Zi1] F. Ziltener, The invariant symplectic action and decay for vortices, J. Symplectic Geom. 7 (3) (2009), 357-376.

[Zi2] F. Ziltener, A Maslov map for coisotropic submanifolds, in preparation.

Department of Mathematics

UNIVERSITY OF TORONTO

40 St. George Street

TORONTO, ONTARIO

CANAdA M5S 2E4

E-mail address: fabian@math.toronto.edu

Received 3/11/2009, accepted 08/14/2009

I am specially indebted to Yael Karshon for numerous enlightening discussions and her continuous support and encouragement. She made me aware that the conclusion of Corollary 1.5 follows from an easy cohomological argument if $(M, \omega):=\left(\mathbb{R}^{2 n}, \omega_{0}\right)$ and $\left(M^{\prime}, \omega^{\prime}\right):=\left(X^{\prime} \times F, \sigma^{\prime} \oplus 0\right)$, with $X^{\prime}$ closed, $\operatorname{dim} X^{\prime}>0$ and $\sigma^{\prime}$ symplectic. I would also like to thank Viktor Ginzburg, Chris Woodward and Masrour Zoghi for interesting conversations, Urs Frauenfelder for useful hints and Andreas Ott for meticulous proofreading. It was Chris Woodward from whom I learned about the construction of the Lagrangian submanifold $\widetilde{N} \subseteq \widetilde{M}$ in the case of a Hamiltonian Lie group action, with $N:=\mu^{-1}(0)$. Finally I would like to thank the referee for valuable suggestions. 